\title{
Numerical modeling of heat transfer and fluid flow in rotor-stator cavities with throughflow
}

\author{
S. Poncet ${ }^{1}$ R. Schiestel ${ }^{2}$ \\ I.R.P.H.E., UMR 6594, CNRS - Universités d'Aix-Marseille I \& II \\ Technopôle Château-Gombert, 49, rue F. Joliot-Curie, 13384 Marseille cédex 13
}

\begin{abstract}
The present study considers the numerical modeling of the turbulent flow in a rotor-stator cavity subjected to a superimposed throughflow with heat transfer. Numerical predictions based on one-point statistical modeling using a low Reynolds number second-order full stress transport closure are compared with experimental data available in the literature $[1,2,3]$. Considering small temperature differences, density variations can be here neglected which leads to dissociate the dynamical effects from the heat transfer process. The fluid flow in an enclosed disk system with axial throughflow is well predicted compared to the velocity measurements performed at IRPHE [3] under isothermal conditions. When the shroud is heated, the effects of rotation and coolant outward throughflow on the heat transfer have been investigated and the numerical results are found to be in good agreement with the data of Sparrow and Goldstein [1]. Their results have been extended for a wide range of the Prandtl numbers. We have also considered the case of an open rotorstator cavity with a radial inward throughflow with heat transfer along the stator, which corresponds to the experiment of Djaoui et al. [2]. Our results have been compared to both their temperature measurements and their asymptotic model with a close agreement between the different approaches, showing the efficiency of the second order modeling. An empirical correlation law is given to predict the averaged Nusselt number depending on the Reynolds and Prandtl numbers and on the coolant flowrate.
\end{abstract}

Key words: rotor-stator system, heat transfer, turbulence modeling

$\overline{1}$ poncet@irphe.univ-mrs.fr - Tel.33 (4) 96.13.97.75

2 schieste@irphe.univ-mrs.fr - Tel.33 (4) 96.13.97.65 


\section{Introduction}

Fluid flow and heat transfer in rotor-stator systems have been the subject of an intense interest, mostly for high-speed rotating gas turbine engines, where air flow in the space between turbine disk and stationary casing is used for cooling purposes. The cooling air is used to both cooling the disk and preventing the ingestion of hot turbine passage gases into the cavity. Nevertheless, many of the published works to date have dealt with the fluid flow aspects of enclosed rotating disks, such as Daily and Nece [4] or open cavities with throughflow, such as Poncet et al. $[3,5]$. The acquisition of heat transfer information has been comparatively slow due to the complexity and the cost of making heat transfer measurements. The objective is to acquire precise knowledge of both the flow structure and the temperature distribution on turbine disks in order to predict durability and to determine the disk dimensions. In the absence of information about local convection, an excessive amount of coolant is often supplied to the rotor-stator cavity, imposing an unnecessary penalty on the engine cycle that must compress and pump the coolant and leads so to a loss of efficiency.

Numerous experimental studies have been carried out since 1960. Nikitenko [6] conducted experiments with an air-filled enclosed rotor-stator system where both disks were isothermal for a wide range of the aspect ratio $0.018 \leq G=$ $h / R_{2} \leq 0.085$ and of the Reynolds number $R e=\Omega R_{2}^{2} / \nu \leq 10^{6}$. We have denoted $h$ the interdisk space, $R_{2}$ the rotating disk radius, $\Omega$ the rate of rotation of the rotor and $\nu$ the kinematic viscosity of the fluid. He found no effect of the aspect ratio and correlated his results according to $N u_{r}=0.0217\left(r^{2} R e\right)^{4 / 5}$, with $r$ the radial location and $N u_{r}=r H / k$ the local Nusselt number ( $H$ the heat transfer coefficient and $k$ the thermal conductivity of the fluid). Kapinos [7] performed measurements of the averaged Nusselt number on an air-cooled rotor, over which the temperature profile was approximately parabolic. He compared these results with the solutions of the turbulent momentum-integral equations for the case of a Batchelor flow (two boundary layers separated by a central rotating core) when a radial outflow is superimposed. He assumed a potential flow in the core with a quasi zero tangential velocity component, which is not appropriate to most industrial applications and to Batchelor type of flow. Using the Reynolds analogy, the calculated Nusselt numbers for a closed cavity with no throughflow $Q$ were equal to those for a free disc. For $C_{w}=Q /\left(\nu R_{2}\right) \rightarrow \infty$, the Nusselt number became independent of Re. He showed that the effect of the flow rate coefficient $C_{w}$ become important to determine the Nusselt number for $5 \times 10^{5} \leq R e \leq 4 \times 10^{6}$ and $0.016 \leq G \leq 0.065$. Owen et al. [8] performed experiments where cooling air is supplied in the centre of an adiabatic stator and a parabolic temperature profile is created on the rotor by means of stationary radiant heaters. The heat transfer was calculated both by solving Laplace's equation for the rotor and by measuring temperatures of the cooling air at inlet and outlet, together with the mea- 
sured windage torque of the rotor. For $0.006 \leq G \leq 0.18$, they showed that the averaged Nusselt number $N u_{a v}=R_{2} H_{a v} / k$ increases as $G$ decreases for small aspect ratios and becomes independent of $G$ for large ones. For a given value of $G$, the Nusselt number increases with increasing values of $C_{w}$. For large Reynolds numbers, the results tend to the free-disc case, whereas for smaller values, they become independent of Re. They noticed a good agreement between the experimental and numerical results. Sparrow and Goldstein [1] performed well controlled heat transfer measurements in a rotor-stator system with an axial outward throughflow. They showed in particular the strong influence of the coolant flow rate on the heat transfer coefficient and the relative importance of rotation. Bunker et al. [9] used the transient liquid crystal technique to measure the radial distribution of convective heat transfer coefficient on both rotor and stator disks. They investigated hub injection of coolant over a wide range of the flow control parameters: $2 \times 10^{5} \leq R e \leq 5 \times 10^{5}$, $835 \leq C_{w} \leq 1670,0.025 \leq G \leq 0.15$. Rotor heat transfer exhibits regions of impingement and dominant rotational effect with a transition region between, while stator heat transfer shows flow reattachement and convection regions with evidence of an inner recirculation zone. The authors pointed out that the calculation of the convective heat transfer coefficient did not account for either recirculating flows or rotation of the core fluid in the cavity. Djaoui et al. $[10,2]$ examined the turbulent flow in a rotor-stator cavity of low aspect ratio subjected to a superimposed radial inflow and heat transfer effects. Detailed velocity and Reynolds stresses measurements as well as temperature and temperature-velocity correlations have been carried out using a hot and cold wire anemometry technique. The temperature distribution was specified on the stator and heat transfer coefficient controlled with the help of pellicular fluxmeters. They studied in particular the external peripheral geometry effects and the critical importance of the inlet conditions on the entrainment coefficient $K$ of the fluid defined as the ratio between the tangential velocity of the fluid and that of the disk at the same radius. They focused also on the dependence of the flow structure and heat transfer effects on the Rossby and Reynolds numbers. Comparisons with an asymptotical formulation based on the assumption of inviscid fluid are displayed and in good agreement with the experimental data. They showed that the main effect of the radial inward throughflow is to increase the heat transfer coefficient on the stator. A detailed review of the main experimental studies on heat transfer in rotor-stator systems is given in [11].

The fact that only few experimental data are available in the literature has slowed down the development of advanced heat transfer models. Haynes and Owen [12] extended numerically the radial outflow study in a shrouded rotorstator system to the heat transfer case. Their numerical model accounts accurately for the increase of averaged Nusselt number with decreasing $G$ and increasing $C_{w}$. For large values of $R e$, the results tend towards those for the free disc. For small values of $R e$, the Nusselt numbers are independent of the rotational speed. More recently, some comparisons between experiments and 
CFD simulations have been performed. In particular, Roy et al. [13] have carried out an experimental study of the fluid temperature distribution and the convective heat transfer coefficient distribution on the rotor disk in a rotorstator cavity with both mainstream and secondary air flow present for a wide range of $R e$ and $C_{w}$. They compared their results to numerical simulations using the commercial CFD code Fluent. In the source region close to the axis of the cavity, the effect of the rotor disk speed was weak and that of the secondary air flow rate strong, the heat transfer coefficient increasing with the air flow rate. In the core region, the convective heat transfer coefficient was controlled mainly by the rotor disk speed, especially at high secondary air flow rates. They provided an empirical correlation for the local Nusselt number on the rotor disk in terms of the local Reynolds number $R e_{r}=\Omega r^{2} / \nu$ : $N u_{r}=0.0074 \times R e_{r}^{0.89}$, which is applicable in the core region and radially outer part of the source region. However the numerical simulations fail to mimic the right trends compared to the experimental data. Beretta and Malfa [14] implemented a semi-empirical model, based on mass and angular momentum balances and the Reynolds analogy, to study the adiabatic rotor and isothermal stator conditions. They compared their results with CFD simulations, with a reasonable agreement for the temperature distribution on the rotor surface in the case of a housing open to radial flow through the gap. The major numerical work is the one of Iacovides and Chew [15]. They have used four different models of turbulence to study the convective heat transfer in three axisymmetric rotating disc cavities with throughflow. Three models were based on a zonal modeling approach and one was based on a mixing-length hypothesis. Their numerical predictions were compared to experimental data available in the literature but none of the four models was entirely successful. Nevertheless, considering overall performance, the $k-\epsilon$ model with the one-equation near-wall treatment is preferred. Schiestel et al. [16] have examined the turbulent flow in a rotating cavity with a radial outward throughflow and heat transfer effects. They compared a standard $k-\epsilon$ low-Reynolds number model and a zonal approach using second-order algebraic stress model in the core of the flow. They showed that second-order modeling is necessary to obtain a detailed near-wall treatment. All workers concluded that further experimental but also numerical research is required before a mathematical model can be recommanded with any confidence. Some advanced heat transfer models have been developed, including the one of Abe et al. [17], who proposed an improved version of the model developed by Nagano et al. [18]. It consists of a two-equation heat transfer model, which incorporates essential features of second-order modeling. They introduced the Kolmogorov velocity scale, instead of the friction velocity, to take into account the low Reynolds number effects in the near-wall region and also complex heat transfer fields with flow separation and reattachement. This model has not been yet implemented for rotor-stator flows. We can also mention Craft [19], who has developed advanced transport closures for the turbulent heat fluxes.

As mentioned above, the difficulty and the cost of measurements under severe 
conditions of rotation and temperature do not allow a full description of the mean and turbulent fields. As a consequence, numerical modeling became a valuable tool for predicting flow structure and heat transfer effects in industrial flows. Previous works $[3,5]$ have shown that second-order modeling is the appropriate way to predict the flow structure in a high-speed rotor-stator cavity with axial inward or outward throughflow, which is a necessary step before a full account of thermal effects can be derived. Because of a lack of detailed heat transfer measurements available in the literature, extensive comparisons can not be performed. Nevertheless, our advanced numerical predictions will be compared to known experimental data for the three different geometries studied by Poncet et al. [3, 5] for fluid flow aspects and by Sparrow and Goldstein [1] and Djaoui et al. [10,2] for thermal effects. Considering that density variation effects are not considered here (temperature variations are small), we did not retain a full transport model for the turbulent heat fluxes. Instead, a gradient hypothesis with tensorial diffusivities is used for closure. Then, the influence of the flow and heat transfer control parameters will be considered.

\section{$2 \quad$ Statistical modeling}

\subsection{Geometrical configurations}

Three different rotor-stator systems are considered in the present study as illustrated in figure 1 in order to test various types of temperature conditions. All cavities are composed of a smooth stationary disk (the stator) and of a smooth rotating disk (the rotor). Case A (fig.1a) models the experiment of Sparrow and Goldstein [1]. The fluid, which is free of prerotation, enters the cavity axially through the central opening and leaves it axially at the periphery through the gap between the rotor and the fixed shroud. The axial throughflow is then centrifugal $(Q<0)$. Case B (fig.1b) models the experiment of Djaoui et al. [2]. In their experiment, a central hub is attached to the rotor and no shroud encloses the cavity. The fluid enters the cavity at its periphery radially through the gap $h$ between the rotor and the stator. It leaves the system axially through the opening between the hub and the stator. The throughflow is then centripetal $(Q>0)$. Case C (fig.1c) represents the IRPHE's experiment [3]. In that case, a fixed shroud encloses the cavity and a hub is attached to the rotor. An axial centripetal or centrifugal throughflow can be superimposed.

The geometrical characteristics of all configurations are displayed in table 1 : $R_{1}, R_{2}$ are the inner and outer radii of the rotating disk, $R_{3}$ the outer radius of the cavity, $R_{4}$ its central opening and $h$ the interdisk space. The mean flow is mainly governed by four control parameters: the aspect ratio of the cavity $G$, the curvature parameter $R_{c}$, the rotational Reynolds number $R e$ based on the outer radius of the rotating disk and the flow rate coefficient $C_{w}$ defined 
as follows:

$$
G=\frac{h}{R_{2}} \quad R_{c}=\frac{R_{2}+R_{1}}{R_{2}-R_{1}} \quad R e=\frac{\Omega R_{2}^{2}}{\nu} \quad C_{w}=\frac{Q}{\nu R_{2}}
$$

where $\Omega$ is the rate of rotation of the rotor, $\nu$ the kinematic viscosity of the fluid and $Q$ the superimposed throughflow. $C_{w}=0$ corresponds to a closed cavity. $C_{w}<0$ (resp. $C_{w}>0$ ) denotes the case where a centrifugal (resp. centripetal) throughflow is superimposed.

Concerning the boundary conditions for the temperature field $T$, the walls and the fluid at the inlet are maintained at a constant temperature denoted $T_{0}$. The black areas in figure 1 (the shroud in case A and the stator in case B) are heated at a constant temperature $T_{w}$, with $T_{w}>T_{0}$. Then, we define the heating factor $\kappa=\left(T_{w}-T_{0}\right) /\left(T_{0}+273\right) \simeq G r / R e^{2}$, where $G r$ is the Grashof number based on the reference temperature $T_{0}$ and the local radius $r$. The typical values used in cases $\mathrm{A}$ and $\mathrm{B}$ are presented in table 1 . Note that the flow in case $\mathrm{C}$ is isothermal. The heating factor is generally small enough to make the hypothesis of no density variation as the gravitational effects are small compared to the inertial effects $\left(G r<<R e^{2}\right)$. The relevant parameter to study heat transfer is the local Nusselt number defined as:

$$
N u=\frac{r J_{w}}{\rho C_{p} \sigma\left(T_{w}-T_{0}\right)}
$$

where $r$ is the radial location, $\sigma$ the thermal diffusivity and $J_{w}$ the wall heat flux given by:

$$
J_{w}=-\left.\rho C_{p} \sigma \frac{\partial T}{\partial z}\right|_{w}
$$

with $\rho$ the fluid density, $C_{p}$ the specific heat, $T$ the local mean fluid temperature and $z$ the axial coordinate. We also define the averaged Nusselt number $N u_{a v}$, which is the averaged value of the local Nusselt number $N u$ along the heated surface, defined as followed:

$$
\begin{array}{cc}
N u_{a v}=\frac{1}{2 \pi h} \frac{\int_{0}^{h} J_{w} d z}{\rho C_{p} \sigma\left(T_{w}-T_{0}\right)} & \text { (case } A) \\
N u_{a v}=\frac{1}{\pi\left(R_{3}^{2}-R_{4}^{2}\right)} \frac{\int_{R_{4}}^{R_{3}} r J_{w} d r}{\rho C_{p} \sigma\left(T_{w}-T_{0}\right)} & \text { (case } B)
\end{array}
$$




\subsection{The differential Reynolds Stress Model (RSM)}

The flows studied here present several complexities (high rotation rate, imposed throughflow, wall effects, transition zones, heat transfer), which are severe conditions for turbulence modeling methods [20, 21, 22]. Our approach is based on one-point statistical modeling using a low Reynolds number secondorder full stress transport closure derived from the Launder and Tselepidakis [23] model and sensitized to rotation effects [24, 25]. Previous works [24, 26, 27] have shown that this level of closure was adequate in such flow configurations, while the usual $k-\epsilon$ model, which is blind to any rotation effect presents serious deficiencies. This approach allows for a detailed description of near-wall turbulence and is free from any eddy viscosity hypothesis. In order to obtain confident heat transfer prediction, it is necessary to reach already a good description of the velocity and turbulence fields. The general equation for the Reynolds stress tensor $R_{i j}$ can be written:

$$
\frac{d R_{i j}}{d t}=P_{i j}+D_{i j}+\Phi_{i j}-\epsilon_{i j}+S_{i j}
$$

where $P_{i j}, D_{i j}, \Phi_{i j}$ and $\epsilon_{i j}$ respectively denote the production, diffusion, pressurestrain correlation and dissipation terms. $S_{i j}$ takes into account the implicit effects of rotation on turbulence.

The diffusion term $D_{i j}$ is split into two parts: a turbulent diffusion $D_{i j}^{T}$, which is interpreted as the diffusion due to both velocity and pressure fluctuations [28] and a viscous diffusion $D_{i j}^{\nu}$, which can not be neglected in the low-Reynolds number region:

$$
\begin{array}{r}
D_{i j}^{T}=\left(0.22 \frac{k}{\varepsilon} R_{k l} R_{i j, l}\right)_{, k} \\
D_{i j}^{\nu}=-\nu R_{i j, k k}
\end{array}
$$

In a classical way, the pressure-strain correlation term $\Phi_{i j}$ can be decomposed as below:

$$
\Phi_{i j}=\Phi_{i j}^{(1)}+\Phi_{i j}^{(2)}+\Phi_{i j}^{(w)}
$$

$\Phi_{i j}^{(1)}$ is interpreted as a slow nonlinear return to isotropy and is modeled as a quadratic development in the stress anisotropy tensor, with coefficients sensitized to the invariants of anisotropy. This term is damped near the wall:

$$
\Phi_{i j}^{(1)}=-\left(\tilde{c_{1}} a_{i j}+c_{1}^{\prime}\left(a_{i k} a_{k j}-\frac{1}{3} A_{2} \delta_{i j}\right)\right) \varepsilon
$$


where $a_{i j}$ denotes the stress anisotropy tensor and $\tilde{c_{1}}$ and $c_{1}^{\prime}$ are two functions deduced from Craft's high-Reynolds number proposals [19] adapted for confined flows:

$$
\begin{aligned}
a_{i j} & =\frac{R_{i j}}{k}-\frac{2}{3} \delta_{i j} \\
\tilde{c_{1}} & =\left(3.1 \sqrt{A A_{2}}+1\right)\left(1-e^{-\frac{R e_{t}^{2}}{40}}\right) \\
c_{1}^{\prime} & =3.72 \sqrt{A A_{2}}\left(1-e^{-\frac{R e_{t}^{2}}{40}}\right)
\end{aligned}
$$

Here $A=1-9 / 8\left(A_{2}-A_{3}\right)$ is the Lumley flatness parameter with $A_{2}$ and $A_{3}$ the second and third invariants of the anisotropy tensor. $R e_{t}=k^{2} /(\nu \varepsilon)$ is the turbulent Reynolds number.

The linear rapid part $\Phi_{i j}^{(2)}$ includes cubic terms. It can be written as:

$$
\begin{aligned}
\Phi_{i j}^{(2)} & =-0.6\left(P_{i j}-\frac{1}{3} P_{k k} \delta_{i j}\right)+0.3 \varepsilon a_{i j} \frac{P_{k k}}{\varepsilon} \\
& -0.2\left[\frac{R_{k j} R_{l i}}{k}\left(V_{k, l}+V_{l, k}\right)-\frac{R_{l k}}{k}\left(R_{i k}\left(V_{j, l}+\epsilon_{j m l} \Omega_{m}\right)\right.\right. \\
& \left.\left.+R_{j k}\left(V_{i, l}+\epsilon_{i m l} \Omega_{m}\right)\right)\right]-\min (0.6, A)\left(A_{2}\left(P_{i j}-\mathcal{D}_{i j}\right)\right. \\
& \left.+3 a_{m i} a_{n j}\left(P_{m n}-\mathcal{D}_{m n}\right)\right)
\end{aligned}
$$

with $P_{i j}=-R_{i j} V_{j, k}-R_{j k} V_{i, k}$ and $\mathcal{D}_{i j}=-R_{i k} V_{k, j}-R_{j k} V_{k, i}$.

Since the slow part of the pressure-strain correlation is already damped near the wall, a wall correction $\Phi_{i j}^{(w)}$ is only applied to the rapid part. The form retained here is the one proposed by Gibson and Launder [29] with a strongly reduced numerical coefficient. Moreover the classical length scale $k^{3 / 2} \varepsilon^{-1}$ is replaced by $k / \varepsilon\left(R_{i j} n_{i} n_{j}\right)^{1 / 2}$ which is the length scale of the fluctuations normal to the wall:

$$
\begin{aligned}
\Phi_{i j}^{(w)}= & 0.2\left[\left(\Phi_{k m}^{(2)}+\Phi_{k m}^{(R)}\right) n_{k} n_{m} \delta_{i j}-\frac{3}{2}\left(\Phi_{i k}^{(2)}+\Phi_{i k}^{(R)}\right) n_{k} n_{j}\right. \\
& \left.-\frac{3}{2}\left(\Phi_{k j}^{(2)}+\Phi_{k j}^{(R)}\right) n_{i} n_{k}\right] \frac{k \sqrt{R_{p q} n_{p} n_{q}}}{\varepsilon y}
\end{aligned}
$$

where $y$ is evaluated by the minimal distance of the current point from the four walls.

The viscous dissipation tensor $\varepsilon_{i j}$ has been modeled in order to conform with 
the wall limits obtained from Taylor series expansions of the fluctuating velocities [30]:

$$
\varepsilon_{i j}=f_{A} \varepsilon_{i j}^{*}+\left(1-f_{A}\right)\left[f_{s} \frac{\varepsilon R_{i j}}{k}+\frac{2}{3}\left(1-f_{s}\right) \varepsilon \delta_{i j}\right]
$$

with $f_{A}, f_{s}$ and $\varepsilon_{i j}^{*}$ defined as followed:

$$
\begin{aligned}
f_{A} & =e^{-20 A^{2}} e^{-\frac{R e_{t}^{2}}{20}} \\
f_{s} & =e^{-\frac{R e_{t}^{2}}{40}} \\
\varepsilon_{i j}^{*} & =\frac{\left(R_{i j}+R_{i k} n_{j} n_{k}+R_{j k} n_{i} n_{k}+R_{k l} n_{k} n_{l} n_{i} n_{j}\right)}{\frac{k}{\varepsilon}\left(1+\frac{3}{2} \frac{R_{p q}}{k} n_{p} n_{q}\right)}
\end{aligned}
$$

The extra term $S_{i j}$ accounts for implicit effects of rotation on turbulence. It contains additional contributions in the pressure-strain correlation, a spectral jamming term, inhomogeneous effects and inverse flux due to rotation, which impedes the energy cascade. This term allowed some improvements of results [24] in the Itoh et al. [31] calculation. These additional terms are fully described in [27].

Below is the proposal of Launder and Tselepidakis [23] for the dissipation rate equation $\varepsilon$ :

$$
\begin{aligned}
\frac{d \varepsilon}{d t}= & -c_{\varepsilon_{1}} \frac{\varepsilon}{k} R_{i j} V_{i, j}-c_{\varepsilon_{2}} f_{\varepsilon} \frac{\tilde{\varepsilon} \varepsilon}{k}+\left(c_{\varepsilon} \frac{k}{\varepsilon} R_{i j} \varepsilon_{, j}+\nu \varepsilon_{, i}\right)_{, i} \\
& +c_{\varepsilon_{3}} \nu \frac{k}{\varepsilon} R_{j k} V_{i, j l} V_{i, k l}+\left(c_{\varepsilon_{4}} \nu \frac{\tilde{\varepsilon}}{k} k_{, i}\right)_{, i}
\end{aligned}
$$

$\tilde{\varepsilon}$ is the isotropic part of the dissipation rate $\tilde{\varepsilon}=\varepsilon-2 \nu k_{i}^{1 / 2} k_{, i}^{1 / 2} \cdot c_{\varepsilon_{1}}=1$, $c_{\varepsilon_{2}}=1.92, c_{\varepsilon}=0.15, c_{\varepsilon_{3}}=2, c_{\varepsilon_{4}}=0.92$ are four empirical constants and $f_{\varepsilon}$ is defined by: $f_{\varepsilon}=1 /\left(1+0.63 \sqrt{A A_{2}}\right)$.

The turbulent kinetic energy equation is redundant in a RSM model but it is however still solved numerically in order to get faster convergence:

$$
\frac{d k}{d t}=-R_{i j} V_{i, j}-\varepsilon+\frac{S_{j j}}{2}+0.22\left(\frac{k}{\varepsilon} R_{i j} k_{, j}+\nu k_{, i}\right)_{, i}
$$

It is verified after convergence that $k$ is exactly $0.5 R_{j j}$. 


\subsection{Thermal field}

As a first approach, we considered only relatively small differences between the temperature of the inlet fluid $T_{0}$ and the wall temperature $T_{w}$. As a consequence, density is not significantly affected by temperature differences. Then, the temperature equation writes:

$$
\frac{\partial T}{\partial t}+V_{j} T_{, j}=\sigma T_{, j j}-F_{j, j}^{t}
$$

where $\sigma=\nu / \operatorname{Pr}(\operatorname{Pr}$ the molecular Prandtl number $)$ and $F_{i}^{t}$ is the turbulent flux approximated by a gradient hypothesis with tensorial diffusive coefficient:

$$
F_{i}^{t}=-c_{t} \frac{k}{\varepsilon} R_{i j} T_{, j}
$$

where $c_{t}=c_{\mu} / P r_{t}=0.1$ with $c_{\mu}=\nu_{t} \varepsilon / k^{2}=0.09$ a coefficient used to define the turbulent viscosity $\nu_{t}$ and $P r_{t}$ the turbulent Prandtl number assumed to be constant at 0.9 [15].

The effects of the anisotropy of the turbulence field and the effects of rotation are already included in $k R_{i j} / \varepsilon$ for most of them.

\subsection{Numerical method}

The computational procedure is based on a finite volume method using staggered grids for mean velocity components with axisymmetry hypothesis in the mean. The computer code is steady elliptic and the numerical solution proceeds iteratively. A $140 \times 80$ mesh in the $(r, z)$ frame proved to be sufficient in cases $\mathrm{B}$ and $\mathrm{C}$ to get grid-independent solutions [26, 27]. A more refined mesh $140 \times 100$ in the axial direction is necessary however in case A in consideration of the aspect ratio. The calculation is initialized using realistic data fields, which satisfy the boundary conditions. About 20000 iterations (several hours on the NEC SX-5, IDRIS, Orsay, France) are necessary to obtain the numerical convergence of the calculation. In order to overcome stability problems, several stabilizing techniques have been introduced in the numerical procedure: diffusive formulation for the Reynolds stress tensor components deduced from the discretized stress equations and reported into the momentum equations [32], implicit treatment of Coriolis type terms and regular and inertial relaxations. Also, the stress component equations are solved using matrix block tridiagonal solution to enhance stability using non staggered grids. 


\subsection{Boundary conditions}

At the wall, all the variables are set to zero except for the tangential velocity $V_{\theta}$, which is set to $\Omega r$ on rotating walls and zero on stationary walls. At the inlet, $V_{\theta}$ is supposed to vary linearly from zero on the stationary wall up to $\Omega r$ on the rotating wall. When a throughflow is enforced, a parabolic profile is then imposed for the axial velocity $V_{z}$, with a given low level of turbulence intensity. In the outflow section, the pressure is fixed, whereas the derivatives for all the other independent quantities are set to zero if the fluid leaves the cavity, and fixed external values are imposed if the fluid re-enters the cavity (as in case C, fig.2c). The continuity equation is then used to determine the outward velocity component. In this case, the boundary condition is of mixed type and a special technique is used to enhance stability [3, 27].

The temperature is set to $T_{0}$ for isothermal walls and at the inlet and to $T_{w}$ for heated walls. A regularization is introduced at the periphery of the stator in case $\mathrm{B}$ as in the experiment of Djaoui et al. [10, 2], where the stator is maintained at $T_{w}$ for $r \leq 0.88 R_{2}$. Another regularization is introduced at the corner between the stator and the shroud in case A in order to stabilize the calculation.

The flow in the similarity area is practically not sensitive to the shape of profiles of tangential and axial velocity components or to the intensity level imposed at the inlet [27]. Moreover, these choices are justified by the wish to have a model as universal as possible. More details about the RSM model and the numerical method are given in [26, 27].

\section{Results and discussion}

\subsection{Fluid flow}

In the present section, we compare the predictions of the RSM model with velocity measurements performed at IRPHE (case C) by the means of a twocomponent laser Doppler anemometer for isothermal turbulent rotor-stator flows. The aim is first to validate our numerical approach from a hydrodynamical point of view. We define the following dimensionless quantities: the radial $r^{*}=r / R_{3}$ and axial $z^{*}=z / h$ coordinates and the mean radial $V_{r}^{*}=V_{r} /(\Omega r)$ and tangential $V_{\theta}^{*}=V_{\theta} /(\Omega r)$ velocity components. Note that $z^{*}=0$ corresponds to the rotor side and $z^{*}=1$ to the stator side.

Figure $2 \mathrm{~b}$ shows the structure of the mean flow in a closed cavity for $R e=$ $1.04 \times 10^{6}$ at $r^{*}=0.56$. The flow is of Batchelor type. It is indeed clearly divided into three distinct zones: a centripetal boundary layer on the stator (Bödewadt layer), a central rotating core and a centrifugal boundary layer on 
the rotor (Ekman or Von Kármán layer). In the Bödewadt layer, the mean radial velocity is negative and the mean tangential velocity ranges between 0 and $K \Omega r$, with $K=0.44$ the entrainment coefficient of the fluid. The central core is characterized by a quasi zero radial velocity and a constant tangential velocity $K \Omega r$. The Ekman layer is always centrifugal: the mean radial velocity is positive and the mean tangential velocity ranges between $\Omega r$ and $K \Omega r$.

When an inward throughflow $C_{w}=5929$ is superimposed (fig.2c), a stagnation line is created on the rotor: the Ekman layer disappears and the core rotates at the same velocity as the rotating disk. It is similar to that observed by Iacovides and Theofanopoulos [33] in the case of a radial centripetal throughflow $C_{w}=3795$ for $R e=6.9 \times 10^{5}, G=0.0685$ and $r^{*}=0.47$.

On the contrary, when a centrifugal throughflow $C_{w}=-5929$ is superimposed (fig.2a), the structure of the flow gets of Stewartson type. It is composed of a single boundary layer on the rotor and a quasi zero tangential velocity outside. The flow is then fully centrifugal $\left(V_{\theta}^{*} \simeq 0\right.$ and $V_{r}^{*}>0$ everywhere) whatever the axial location $z^{*}$. Note that the profile of the radial velocity is asymmetric showing the effect of the centrifugal force on the rotating disk.

Comparisons for three components of the Reynolds stress tensor are given in figure 3 for $R e=1.04 \times 10^{6}$ and three flow rate coefficients at $r^{*}=0.56$ : two normal components $R_{r r}^{*}=\overline{v_{r}^{\prime 2}} /(\Omega r)^{2}, R_{\theta \theta}^{*}=\overline{v_{\theta}^{\prime 2}} /(\Omega r)^{2}$ and one shear component $R_{r \theta}^{*}=\overline{v_{r}^{\prime} v_{\theta}^{\prime}} /(\Omega r)^{2}$. In the case of a closed cavity (fig.3b), the turbulence intensities are rather weak and mostly concentrated in the boundary layers. The Ekman layer is besides more turbulent than the Bödewadt layer. In the rotating core, the Reynolds stresses are weak. $R_{r \theta}^{*}$ is indeed almost zero, that means that there is no turbulent shear stress in that zone. At this radial location $r^{*}=0.56$, some discrepancies occur mainly in the low turbulence intensity regions where relaminarization is expected.

Figure $3 \mathrm{c}$ shows the effect of a centripetal throughflow on the turbulence intensities for $C_{w}=5929$. The Bödewadt layer is more turbulent than the Ekman layer. The viscous shear stress is quasi zero in this layer, whereas it is large on the stator. The value of the normal Reynolds stress tensor components are quite comparable at this radial location. The Reynolds stress tensor components are well predicted apart from the $R_{r \theta}^{*}$ component, whose absolute magnitude is overestimated in the Bödewadt layer.

Figure 3a exhibits the axial profiles of three Reynolds stress components, when a centrifugal throughflow is superimposed $C_{w}=-5929$. Contrary to the case with a centripetal throughflow, the maxima of the turbulence intensities are confined in the Ekman layer and they vanish in the Bödewadt layer. As a conclusion, turbulence is mainly governed by the axial throughflow and not by rotation, which has been shown by [27].

We recall that the aim of this section was not to discuss about the physics of rotating disk flows but to validate the performances of the RSM model. Poncet et al. [3] have already performed a parametric study of the turbulent flow in a rotor-stator cavity when an inward or outward throughflow is superimposed. 
Here, the RSM predictions are in very good agreement with the experimental data, even in the boundary layers, for the mean and turbulent fields (fig.2 and 3) for all considered cases. It proves that the RSM model is the adequate level of closure to describe all type of rotating disk flows: Batchelor or Stewartson flow structures.

\subsection{Temperature field and heat transfer properties}

\subsubsection{Case $A$}

We first consider the cavity sketched in figure 1a, which models the experiment of Sparrow and Goldstein [1]. We recall that we restricted the calculation to constant wall temperature $T_{w}$ heating of the shroud and that these authors do not performed any measurements of the fluid flow.

Distributions of the local Nusselt number along the shroud are presented in figure 4 for different flow conditions $\left(R e, C_{w}\right)$ and a given Prandtl number $\operatorname{Pr}=0.7$. For all cases, the local Nusselt number decreases with increasing distance from the rotor. This can be explained by looking at the streamline patterns (fig.5), whose structure in the vicinity of the external stationary shroud is rather complex. One can notice near the rotating disk the occurance of a stagnation point at $z^{*} \simeq 0.12$, where the centrifugal flow impinges the external wall. Impinging jets are known to be a difficult situation for turbulence model, in particular in the calculation of the turbulent diffusivity [34] even if the geometry involved here is however somehow different from an usual impinging jet. The radial outflow on the disk, after the impingement on the shroud, is deflected as an axial flow along it in the positive z-direction. As the fluid flows along the shroud, the thermal boundary layer thickens, and as a consequence, the thermal resistance of the flow increases. Close to the stator side, the presence of a weak secondary recirculation zone (fig.5a-b) gives rise to a low velocity region with relatively low heat transfer. This complex configuration may explain one part of the remaining discrepancies observed in figure 4 on the axial variations of the local Nusselt number. It is indeed a difficult case because of the combination of rotation effects and strong curvature of streamlines due to deflection. The effect of the Reynolds number on the local Nusselt number $N u$ is shown in figure 4a for given coolant flowrate coefficient $C_{w}=-2749$ and Prandtl number $\operatorname{Pr}=0.7$. The local Nusselt number increases with increasing values of the Reynolds number. As expected, the heat transfer is enhanced by the rotational disk speed. Nevertheless, the influence of $R e$ is less important than the one of the coolant flowrate coefficient. Sparrow and Goldstein [1] noticed indeed that $N u$ is insensitive to a critical Reynolds number up to $R e_{c}=1.56 \times 10^{5}$ for $C_{w}=-2749$. This value increase for increasing values of $C_{w}$. For example, $R e_{c}=4.65 \times 10^{5}$ for $C_{w}=-4392$ and for $C_{w}=-8672$, the effect of $R e$ is small over the entire range of $R e$ investigated 
here. Nusselt numbers are known to be very sensitive to sublayer modeling. The numerical predictions are here qualitatively in good agreement with the measurements of Sparrow and Goldstein [1] and especially for $R e=9.2 \times 10^{5}$ and $C_{w}=-2749$. In that case, the recirculation area at the periphery of the cavity (fig.5c) is much smaller than in the other cases, that could explain the good agreement as recirculation induces a highest level of complexity to predict heat transfer. On the other hand, on the stator side, where the fluid is at rest (fig.5a and 5b), the local Nusselt number $N u$ is less well predicted and is insensitive to the Reynolds number.

The effect of the coolant flowrate coefficient $C_{w}$ on the local Nusselt number $N u$ is shown in figure $4 \mathrm{~b}$ for given Reynolds $R e=1.56 \times 10^{5}$ and Prandtl $\operatorname{Pr}=0.7$ numbers. As heat transfer is enhanced by convection, the local Nusselt number increases with increasing values of $C_{w}$. The radial outflow due to the outward throughflow adds to the one due to the pumping effect of the rotating disk (the centrifugal force). That induces higher velocities, which make the intensity of the axial flow along the shroud is much stronger and thereby enhances the heat transfer coefficient reflected in the $N u$ values. Moreover, the recirculation area in the core of the flow increases with increasing values of $C_{w}$ (fig.5a,d,e). The effect of $C_{w}$ on the Nusselt number is more important on the rotor side, which may be related to the greater sensitivity of thinner boundary layers to changes in velocity. Sparrow and Goldstein [1] have also investigated the influence of the aspect ratio of the cavity on the local Nusselt number. They concluded that the major effect of decreasing aspect ratio is to bring a general increase in the magnitude of $N u$.

We focus our attention to the temperature field for the same flow conditions $\left(R e, C_{w}\right)$ and a given Prandtl number $\operatorname{Pr}=0.7$. We define the dimensionless temperature $T^{*}=\left(T-T_{0}\right) /\left(T_{w}-T_{0}\right)$. The figure 6 exhibits the temperature profiles along the axial direction for five flow conditions at three radial locations. Whatever the radial location $r^{*}$, the temperature is almost constant in the core of the flow and tends to zero close to the walls. At $r^{*}=0.68$ (fig.6a), $T^{*} \simeq 0.25$ in the core and depends only slightly of the flow parameters $R e$ and $C_{w}$. When one approaches the periphery of the cavity, the influences of the heated shroud, the recirculation area and the axial flow in the Stewartson layer (along the shroud) are more important and the temperature profiles depend on the Reynolds number and the coolant flowrate coefficient. At $r^{*}=0.84$ (fig.6b), the temperature decreases for all flow conditions excepted for $R e=9.2 \times 10^{5}$ and $C_{w}=-2749$. This decrease can be explained by looking at the corresponding isotherm maps (fig.7). We can notice that they are clearly influenced by the recirculation area observed in figure 5 . The flow is then dominated by the outward throughflow and the recirculation area increases with increasing values of the flowrate coefficient. The temperature in these cases may be more influenced by the cold flow coming from the zone close to the axis of the cavity than by the heated shroud. On the contrary, for $R e=9.2 \times 10^{5}$ and $C_{w}=-2749$, the flow is dominated by rotation and no recirculation is observed for $r^{*} \geq 0.6$. The isotherms (fig.7c) are then parallel 
to the axis of rotation and the flow is more sensitive to the heated shroud. At $r^{*}=0.96$ (fig.6c), the temperature profiles look rather the same. The effect of the heated shroud on the temperature field is compensated by the cold fluid flowing in the Ekman layer, impinging on the shroud and then cooling the flow along the shroud. The first noticeable effect is the increase of the temperature in the Bödewadt layer due to the warm inward flow passes in that layer and coming from the area close the heated shroud. We can notice also a bump on $T^{*}$ profiles for $R e=1.56 \times 10^{5}$ and $C_{w}=-2749$. This hot point may be due to the fact that the fluid is trapped (fig.5a) at the periphery of the cavity and continuously heated by the shroud.

We have characterized the influence of the Reynolds number and the coolant flowrate coefficient on the local Nusselt number. The predictions of the RSM model have been found to be in good agreement with the experimental data of Sparrow and Goldstein [1]. The RSM model has been used also to extend the results of these last authors and especially to characterize the effect of the Prandtl number $\operatorname{Pr}$ on the local Nusselt number $N u$. Figure 8 shows the effect of the Prandtl number on the $N u$ distribution along the shroud for $R e=9.2 \times 10^{5}$ and $C_{w}=-2749$. The Prandtl number $\operatorname{Pr}$ can be related to the thickness of the thermal and velocity boundary layers. It is actually the ratio of velocity boundary layer to thermal boundary layer. When $\operatorname{Pr}=1$, the boundary layers more or less coincide. We have tested several values of $\operatorname{Pr}$ in the range $0.01-12$, which is impossible in experimental works. $\operatorname{Pr}=0.01$ is a typical value for liquid metals, $P r=0.7-1$ for gases $(\operatorname{Pr}=0.71$ for air at $20^{\circ} \mathrm{C}$ ) and $\operatorname{Pr}=2-12$ for water. As expected, the local Nusselt number $N u$ increases with increasing values of the Prandtl number $\mathrm{Pr}$. The low value of Nusselt number for the liquid metal range reflects the dominating influence of molecular diffusivity.

From an industrial point of view, the interesting quantity is the averaged Nusselt number $N u_{a v}$. We recall that $N u_{a v}$ is the average of the local Nusselt number along the heated shroud. The effects of the coolant flowrate coefficient $C_{w}$, the Reynolds Re and Prandtl $\operatorname{Pr}$ numbers on the averaged Nusselt number $N u_{a v}$ are summed up in figure $9 . N u_{a v}$ behaves qualitatively like $N u$ : it increases with increasing values of $\operatorname{Pr}, \operatorname{Re}$ or $C_{w}$. Note that for small values of $P r$, the thermal time is larger than the viscous time and so the diffusion process pilots the fluid flow. On the contrary, for large Prandtl numbers, the thermal effects vanish and the flow behavior is essentially hydrodynamic.

As a conclusion, we have studied the fluid flow and the heat transfer in a rotor-stator cavity with an axial outward throughflow and heat transfer along the shroud. Our numerical results have been compared with the experimental data of Sparrow and Goldstein [1] with a good agreement between the two approaches, especially in the case where rotation dominates the flow. When the flow is dominated by the outward throughflow, a large recirculation area appears at the periphery of the cavity and the heat transfer is less well predicted. The discrepancies, which remain, can be attributed partly to experimental uncertainties with inlet and temperature boundary conditions. Warm fluid from 
outside the cavity may be drawn in through the outlet and affect the heat transfer along the shroud. The second explanation may be the relatively important value of $\kappa=0.254$. The temperature difference of $75^{\circ} \mathrm{C}$ can indeed produce density variations that influence the mass conservation equation. The influence of the Reynolds number $R e$ and the coolant flowrate coefficient $C_{w}$ have been characterized. Moreover, the results of Sparrow and Goldstein [1] have been extended for a wide range of Prandtl numbers $\mathrm{Pr}$. The Nusselt numbers $N u$ and $N u_{a v}$ appear to be an increasing function of $R e, C_{w}, \operatorname{Pr}$ and of the axial position.

\subsubsection{Case B}

We consider the fluid flow and the heat transfer in a rotor-stator cavity with a radial inward throughflow and heat transfer along the stator. The cavity is sketched in figure $1 \mathrm{~b}$ and models the experiment of Djaoui et al. [2]. The aspect ratio of the cavity $G$ is fixed to $G=0.08$ and we study the influence of the coolant flowrate coefficient $C_{w}$ and the Reynolds $R e$ and Prandtl $\mathrm{Pr}$ numbers on the local Nusselt number $N u$.

For given flow conditions, variations of $N u$ versus $r^{*}$ are represented in figure 10. In all cases, $N u$ increases with $r^{*}$ essentially because $N u$ is the local Nusselt number based on the radial location $r^{*}$. The strong increase (or decrease for $R e=5 \times 10^{5}$ and $C_{w}=12082$ in figure 10b) of $N u$ observed for $r^{*} \geq 0.75$ is certainly due to the peripheral effects. The variations of $N u$ are in connection with the changes in velocity. We first focus on the influence of the coolant flowrate coefficient $C_{w}$ for $R e=1.44 \times 10^{6}$ and $\operatorname{Pr}=0.7$ (fig.10a). In the case of the isolated cavity $C_{w}=0$, the fluid rotates as a solid body in the whole cavity (fig.11e), the flow is then of Batchelor type with two boundary layers separated by a central rotating core as already reported in figure $2 \mathrm{~b}$ and no secondary recirculation is observed. The isotherm maps (fig.12e) look like the streamline patterns and we can clearly see the two thermal boundary layers developed on each disk. As a consequence, the RSM model predicts very well the local Nusselt number $N u$. By increasing the coolant flowrate coefficient (fig.10a), the flow gets confined in the Bödewadt layer, which thickens (fig.11c,d) and the fluid velocity in that layer increases. So the local Nusselt number $N u$ increases too. We can also observe a large recirculation zone, which appears at the periphery of the cavity for $z^{*} \leq 0.65$ and gets thinner as the inward throughflow gets stronger. For large $r^{*}$, the vortex is expected to play a dominant part as already observed in case A. As $N u$ is not very sensible to $C_{w}$, whereas the size of the vortex changes, it may show that the heat transfer occur essentially in the stator boundary layer. The fluid warms up along the stator, flows through the axis of the cavity and goes out. In the same time, the rotor carries cold fluid through the periphery. For large values of $C_{w}$ (fig.12c), where rotation plays a minor role compared to the coolant throughflow, there is no more convection as observed at the periphery of the 
cavity for weaker throughflow (fig.12b). The isotherms are besides parallel to the two disks. For the three cases considered here, the RSM model predicts quite well the local Nusselt number even if it seems to be less sensible to the effect of $C_{w}$ than the experimental data.

The effect of the Reynolds number $R e$ has also been investigated for $\operatorname{Pr}=0.7$ and $C_{w}=12082$ (fig.10b). For the three values of $R e$ considered here, the effect of the inward throughflow dominates the one of the rotation. As shown in figure 11a-c, the fluid flows through the axis of the cavity along the stator side. A recirculation zone subsists at the periphery but its size decreases with decreasing values of the Reynolds number. Moreover, the tangential component of the fluid velocity decreases as the rate of rotation decreases. Consequently, the local Nusselt number $N u$ decreases with decreasing values of $R e$ (fig.10b). For this strong inward throughflow, whatever the Reynolds number, the isotherms (fig.12) are almost parallel to the disks and no convection is observed. For $R e=5 \times 10^{5}$ (fig.12a), there is a important zone where no variations of $T^{*}$ are observed in a large range of axial locations $z^{*}$, which may explain the decrease of $N u$ in that region (fig.10b).

The figure 13 exhibits some temperature profiles for the particular case $C_{w}=$ 12082, $R e=1.44 \times 10^{6}$ and $\operatorname{Pr}=0.7$ at three radial locations. The predictions of the RSM model are compared to both the theoretical and experimental values obtained by Djaoui et al. [2] for the same case. The predictions of the RSM model are found to be in particular good agreement with their theoretical model. It consists of an asymptotic approach where compressibility, buoyancy and viscous dissipation (outside the boundary layers) can be neglected as $G<<1, \operatorname{Pr} \simeq 1, \kappa<<1,\left(\operatorname{Re} G^{2}\right)^{-1}<<1$ and the Eckert number is small. The asymptotic solution gives: $T^{*}=1-3.8 r^{* 2}$. The agreement is good also with the experimental data in the core of the flow but the measurements are more difficult close to the walls and no information is available in the boundary layers. The temperature profiles look like the tangential velocity ones: $T^{*}$ decrease from 1 to $K_{T}$ in the thermal boundary layer attached to the heated stator, and is almost constant and equal to $K_{T}$ in the core and tends to zero by approaching the rotor. Note that $K_{T}$ is the ratio between the temperature of the heated wall $T_{w}$ and the temperature in the core of the flow. $K_{T}$ increases slightly near the axis of the cavity ranging from 0.22 at $r^{*}=83$ (fig.13c) to 0.26 at $r^{*}=0.53$ (fig.13a). In the same way, the thermal boundary layer attached to the stator thickens with decreasing values of the radial location $r^{*}$.

The RSM model has been used also to extend the results of Djaoui et al. [2] and especially to characterize the effect of the Prandtl number $\mathrm{Pr}$ on the local Nusselt number $N u$. Figure 14 shows the effect of the Prandtl number on the local Nusselt number distribution along the stator for $R e=1.44 \times 10^{6}$ and $C_{w}=12082$. As observed in case A, $N u$ increases with increasing values of the Prandtl number $\operatorname{Pr}$ and the same conclusions can be made.

The effects of the coolant flowrate coefficient $C_{w}$, the Reynolds $R e$ and Prandtl $\operatorname{Pr}$ numbers on the averaged Nusselt number $N u_{a v}$ are summed up in figure 
15a. As for case A, $N u_{a v}$ behaves qualitatively like the local Nusselt number $N u$ : it increases with increasing values of $\operatorname{Pr}, R e$ or $C_{w}$ apart from the case $R e=1.44 \times 10^{6}$ and $C_{w}=12082$, where $N u_{a v}$ is less important than for $R e=1.44 \times 10^{6}$ and $C_{w}=5209$ for Prandtl numbers larger than one. It may be assigned to peripheral effects $\left(r^{*} \leq 0.3\right.$ and $\left.r^{*} \geq 0.85\right)$. Note that for small values of $\mathrm{Pr}$, the thermal time is larger than the viscous time and so the molecular diffusion process pilots the heat transfer. On the contrary, for large Prandtl numbers, the molecular thermal effects vanish and the heat transfer behavior is turbulent. For engineering applications, we searched an empirical correlation for $N u_{a v}$ as a function of $R e, C_{w}$ and $\operatorname{Pr}$, which best fits the numerical predictions. All the numerical results were found to be correlated by the following empirical law:

$$
N u_{a v}=0.0044 \operatorname{Re}^{0.8}\left(1000+C_{w}\right)^{0.11} \operatorname{Pr}^{0.5}
$$

This empirical law (23) has been validated for the entire range of Reynolds numbers $5 \times 10^{5} \leq R e \leq 1.44 \times 10^{6}$ and coolant flowrates $0 \leq C_{w} \leq 12082$ considered here and for $1 \leq P r \leq 12$. The figure $15 \mathrm{~b}$ confirms the dependence of $N u_{a v}$ on the flow parameters. The averaged Nusselt number $N u_{a v}$ depends on the global Reynolds number to the power 0.8, which confirms a lot of previous results, such as those of Nikitenko [6], Yu et al. [35] or Shchukin and Olimpiev [36]. This correlation law can be extended to other values of the aspect ratio $G$ of the cavity, as Owen and Rogers [11] have shown that $N u_{a v}$ is almost constant for $G \geq 0.04$.

The Reynolds analogy has often be used to be compared to experimental data. Using the modified Reynolds analogy $N u_{a v}=R e \times C_{M} \times \operatorname{Pr}^{0.6} / \pi\left(C_{M}\right.$ is the moment coefficient), Owen and Haynes [37] obtained a good agreement with the measurements of Owen et al. [8]. For large coolant flowrate coefficients $\left(C_{w} /(G R e)>10\right)$, the Reynolds analogy gives an asymptotic solution in the form: $N u_{a v} \propto\left(C_{w} / G\right)^{0.8}$ [11]. In our case, $0 \leq C_{w} /(G R e) \leq 3$ but we can suppose that the Reynolds analogy is not verified. Indeed, $N u \propto C_{w}^{0.11}$ far from the power 0.8 prescribed by [11].

To go further, we have also compared the velocity-temperature correlations $F_{r}^{t *}$ and $F_{\theta}^{t *}$ given by the relation (22) and normalized by $\Omega R_{2}\left(T_{w}-T_{0}\right)$ to the measurements of Djaoui et al. [2] for $R e=1.44 \times 10^{6}, \operatorname{Pr}=0.7$ and $C_{w}=$ 12082 at three radial locations (fig.16). Even if some discrepancies are observed and in spite of the use of tensorial thermal diffusivity, some trends can be inferred from these comparisons. The magnitudes of these two turbulent fluxes, which are rather weak, decrease slightly when one approaches the periphery of the cavity. The discrepancies remain essentially because of the poor accuracy of measurements as already noticed by Djaoui et al. [2]. Moreover, as their magnitudes are quite weak, their influence may be small too, because the flow is mainly governed by the viscous diffusion instead of the turbulent one.

So, in case B, we have studied the fluid flow and the heat transfer in a rotor- 
stator cavity with an radial inward throughflow and heat transfer along the fixed disk. Our numerical results have been compared with the experimental and theoretical data of Djaoui et al. [2] with a good agreement between the different approaches, especially in the case in which rotation dominates the flow. The flow is purely centripetal and essentially confined in the Bödewadt layer along the stator. This can be considered as a kind of flow over a plane plate, for which it is well known that heat transfer is well predicted. This configuration is then favourable to predict heat transfer in rotor-stator systems than case A. The influence of the coolant flowrate coefficient $C_{w}$ has been characterized but we have also extended the results of Djaoui et al. [2] for different Reynolds numbers $R e$ and for a wide range of Prandtl numbers $\operatorname{Pr}$. The Nusselt numbers $N u$ and $N u_{a v}$ appear to be an increasing function of $R e, C_{w}, \operatorname{Pr}$ and of the radial position.

\section{Conclusion}

We have proposed some calculations of the turbulent flow inside three different rotor-stator systems with superimposed throughflow and heat transfer. These configurations are especially representative of those encountered in gas-turbine engines. The predictions of a second order model have been compared to experimental data available in the literature $[1,2,3]$. The RSM has first proved his efficiency, compared to the velocity measurements performed at IRPHE [3], to predict the turbulent flow inside a rotor-stator cavity with axial inward or outward throughflow, under isothermal conditions. This was a prerequisite for studying heat transfer. It has then been used to study the heat transfer inside two different configurations, for which well controlled experiments [1, 2] have been performed. As a first step, the variations of density have been neglected allowing to dissociate the dynamical effects from the heat transfer process.

We have first investigated the effects of rotation and coolant outward throughflow on the heat transfer in a rotor-stator cavity enclosed by a heated shroud and subjected to an axial outward throughflow, corresponding to the case studied experimentally by Sparrow and Goldstein [1]. The numerical results are found to be in satisfactory agreement with their experimental data. Some discrepancies remain essentially because of the temperature difference between the heating temperature of the shroud and the inlet temperature of the fluid is not small enough making the hypothesis of no density variation somewhat questionable. Moreover, the heat transfer is dominated by a large recirculation zone, which appears at the periphery of the cavity and, which is a penalizing configuration for numerical modeling. Their results have also been extended numerically for a wide range of Prandtl numbers. The local Nusselt number increases with increasing values of the Reynolds and Prandtl numbers and of the coolant flowrate. It decreases when one approaches the stator side. 
The cavity studied by Djaoui et al. [2] has been also considered. It consists of a rotor-stator system where the stator is heated at a constant temperature and a radial inward throughflow is superimposed. Our results have been compared to both their temperature measurements and their theoretical approach. They are in closer agreement showing the efficiency of the second order model. Here again, the effects of the Reynolds number and the coolant flowrate coefficient on the local Nusselt number $N u$ have been studied and, as in the previous case, the same conclusions arise. The main differences, which can explain this closer agreement, are firstly the open geometry of the cavity, in which the inward throughflow does not impinge on any wall. Secondly, the secondary recirculation zone is smaller and does not appear close to the heated wall but along the rotor side and may affect not as efficiently as in the previous case. The RSM model allowed a close agreement with experiments for three different configurations and so can be recommanded for engineering predictions. Nevertheless, further numerical work is now likely required to take into account the important temperature differences, which are often encountered in gas-turbine engines. Density variation effects have then to be considered. But further experimental research is also suitable to extend the parameter range for more comparisons and especially concerning the effect of the Prandtl number.

\section{Acknowledgement}

Numerical computations have been carried out on the NEC SX-5 at the IDRIS (Orsay, France), who is gratefully acknowledged.

\section{References}

[1] E.M. Sparrow and J.L. Goldstein. Effect of rotation and coolant throughflow on the heat transfer and temperature field in an enclosure. J. Heat Transfer, 98:387-394, 1976.

[2] M. Djaoui, A. Dyment, and R. Debuchy. Heat transfer in a rotor-stator system with a radial inflow. Eur. J. Mech. B - Fluids, 20:371-398, 2001.

[3] S. Poncet, M.P. Chauve, and R. Schiestel. Batchelor versus Stewartson flow structures in a rotor-stator cavity with throughflow. Phys. Fluids, 17(7), 2005.

[4] J.W. Daily and R.E. Nece. Chamber dimension effects on induced flow and frictional resistance of enclosed rotating disks. ASME J. Basic Eng., 82:217-232, 1960.

[5] S. Poncet, R. Schiestel, and M.P. Chauve. Centrifugal flow in a rotorstator cavity. J. Fluid. Eng., 127(4):687-694, 2005. 
[6] N.I. Nikitenko. Experimental investigation of heat exchange of a disk and a screen. J. Engng Phys., 6:1-11, 1963.

[7] V.M. Kapinos. Heat transfer from a disc rotating in a housing with a radial flow of coolant. J. Engng Phys., 8:35-38, 1965.

[8] J. M. Owen, C.M. Haynes, and F.J. Bayley. Heat transfer from an aircooled rotating disk. Proc. Roy. Soc. A, 336:453-473, 1974.

[9] R.S. Bunker, D.E. Metzger, and S. Wittig. Local heat transfer in turbine disk-cavities - part.1: Rotor and stator cooling with hub injection of coolant. ASME J. Turbomach., 114:211-220, 1990.

[10] M. Djaoui and R. Debuchy. Heat transfer in a rotor-stator system with a radial inflow. C. R. Acad. Sci. Paris IIb, 326:309-314, 1998.

[11] J. M. Owen and R. H. Rogers. Flow and Heat Transfer in Rotating-Disc Systems - Vol.1: Rotor-Stator Systems. Ed. Morris, W.D. John Wiley and Sons Inc., New-York, 1989.

[12] C.M. Haynes and J.M. Owen. Heat transfer from a shrouded disk system with a radial outflow of coolant. J. Engng for Power, 97:28-36, 1975.

[13] R.P. Roy, G. Xu, and J. Feng. A study of convective heat transfer in a model rotor-stator disk cavity. ASME J. Turbomach., 123:621-632, 2001.

[14] G.P. Beretta and E. Malfa. Flow and heat transfer in cavities between rotor and stator disks. Int. J. Heat Mass Transfer, 46:2715-2726, 2003.

[15] H. Iacovides and J.W. Chew. The computation of convective heat transfer in rotating cavities. Int. J. Heat Fluid Flow, 14(2):146-154, 1992.

[16] R. Schiestel, L. Elena, and T. Rezoug. Numerical modeling of turbulent flow and heat transfer in rotating cavities. Numerical Heat Transfer A, 24:45-65, 1993.

[17] K. Abe, T. Kondoh, and Y. Nagano. A two-equation heat transfer model reflecting second-moment closures for wall and free turbulent flows. Int. J. Heat Fluid Flow, 17:228-237, 1996.

[18] Y. Nagano, M. Tagawa, and T. Tsuji. An improved two-equation heat transfer model for wall turbulent shear flows. pages 233-240, New-York, 1991. Proc. of the ASME/JSME Thermal Engineering Joint Conference - Vol.3, Ed. J. Lloyd and Y. Kurosaki, ASME.

[19] T.J. Craft. Second-moment modelling of turbulent scalar transport. PhD thesis, University of Manchester, 1991.

[20] P. Bradshaw. The analogy between the streamline curvature and buoyancy in turbulent shear flow. J. Fluid. Mech., 36:177-191, 1969.

[21] C. Cambon and L. Jacquin. Spectral approach to non isotropic turbulence subjected to rotation. J. Fluid. Mech., 202:295-317, 1989.

[22] L. Jacquin, O. Leuchter, C. Cambon, and J. Mathieu. Homogeneous turbulence in the presence of rotation. J. Fluid. Mech., 220:1-52, 1990.

[23] B.E. Launder and D.P. Tselepidakis. Application of a new secondmoment closure to turbulent channel flow rotating in orthogonal mode. Int. J. Heat Fluid Flow, 15(1):2-10, 1994.

[24] L. Elena and R. Schiestel. Turbulence modeling of rotating confined flows. Int. J. Heat Fluid Flow, 17:283-289, 1996. 
[25] R. Schiestel and L. Elena. Modeling of anisotropic turbulence in rapid rotation. Aerospace Science and Technology, 7:441-451, 1997.

[26] L. Elena. Modélisation de la turbulence inhomogène en présence de rotation. PhD thesis, Université Aix-Marseille I-II, 1994.

[27] S. Poncet. Écoulements de type rotor-stator soumis à un flux: de Batchelor à Stewartson. PhD thesis, Université Aix-Marseille I, 2005.

[28] B.J. Daly and F.H. Harlow. Transport equation for turbulence. Phys. Fluids A, 13(11):2634-2649, 1970.

[29] M. Gibson and B.E. Launder. Ground effects on pressure fluctuations in the atmospheric boundary layer. J. Fluid. Mech., 86(3):491-511, 1978.

[30] B.E. Launder and W.C. Reynolds. Asymptotic near-wall stress dissipation rates in a turbulent flow. Phys. Fluids A, 26(5):1157-1158, 1983.

[31] M. Itoh, Y. Yamada, S. Imao, and M. Gonda. Experiments on turbulent flow due to an enclosed rotating disk. pages 659-668, New-York, 1990. Proc. Int. Symp. on Engineering Turbulence Modeling and Experiments, Ed. W. Rodi and E.N. Ganic, Elsevier.

[32] P.G. Huang and M.A. Leschziner. Stabilization of recirculating flow computations performed with second moments closures and third order discretization. Cornell University, Ithaca, NY, 1985. Vth Int. Symp. on Turbulent Shear Flow.

[33] H. Iacovides and I.P. Theofanopoulos. Turbulence modeling of axisymmetric flow inside rotating cavities. 12(1):2-11, 1991.

[34] C. Yap. Turbulent heat momentum transfer in recirculating and impinging flows. PhD thesis, Dpt Mech. Engng, University of Manchester, 1987.

[35] J.P. Yu, E.M. Sparrow, and E.R.G. Eckert. Experiments on a shrouded, parallel disk system with rotation and coolant throughflow. Int. J. Heat Mass Transfer, 16:311-328, 1973.

[36] V.K. Shchukin and V.V. Olimpiev. Heat transfer of disc rotating in a housing with transitional and turbulent boundary layers. Soviet Aeronautics, 18:77-81, 1975.

[37] J. M. Owen and C.M. Haynes. Design formulae for the heat loss and frictional resistance of air-cooled rotating discs. pages 127-160, Milan, 1976. Improvements in Fluid Mechanics and Systems for Energy Conversion Vol.4, Ed. Hoepli. 
- Fig.1: Sketch of the three considered cavities and relevant notation.

- Fig.2: Mean velocity profiles in case $\mathrm{C}$ at $r^{*}=0.56$ for $R e=1.04 \times 10^{6}$ and three flow rate coefficients: (a) $C_{w}=-5929$, (b) $C_{w}=0$, (c) $C_{w}=5929$; Comparisons between (o) velocity measurements and (-) the RSM model.

- Fig.3: Axial variations of three Reynolds stress tensor components in case $\mathrm{C}$ at $r^{*}=0.56$ for $R e=1.04 \times 10^{6}$ and three flow rate coefficients: (a) $C_{w}=-5929,(\mathrm{~b}) C_{w}=0$, (c) $C_{w}=5929$; Comparisons between (o) velocity measurements and (-) the RSM model.

- Fig.4: Local Nusselt number distributions along the shroud $\left(r^{*}=1\right)$ (case A): (a) effect of the Reynolds number for $C_{w}=-2749$ and $\operatorname{Pr}=0.7$, (b) effect of the flow rate coefficient for $R e=1.56 \times 10^{5}$ and $\operatorname{Pr}=0.7$. Comparisons between (symbols) the measurements of Sparrow and Goldstein [1] (SG) and (lines) the predictions of the RSM model.

- Fig.5: Streamlines $\Psi^{*}=\Psi /\left(\Omega R_{2}^{2}\right)$ patterns (RSM), 30 regularly spaced intervals: (a) $R e=1.56 \times 10^{5}, C_{w}=-2749,-0.001 \leq \Psi^{*} \leq 0.032$; (b) $R e=4.65 \times 10^{5}, C_{w}=-2749,-0.001 \leq \Psi^{*} \leq 0.026$; (c) $R e=9.2 \times 10^{5}$, $C_{w}=-2749,-0.002 \leq \Psi^{*} \leq 0.009 ;$ (d) $R e=1.56 \times 10^{5}, C_{w}=-4392$, $-0.002 \leq \Psi^{*} \leq 0.101 ;(\mathrm{e}) R e=1.56 \times 10^{5}, C_{w}=-8672,-0.005 \leq \Psi^{*} \leq$ 0.263 .

- Fig.6: Temperature profiles for different flow conditions $\left(R e, C_{w}\right)$ at three radial locations: (a) $r^{*}=0.68$, (b) $r^{*}=0.84$, (c) $r^{*}=0.96$ (RSM).

- Fig.7: Isotherm maps $T^{*}$ (RSM), 30 regularly spaced intervals: (a) $R e=$ $1.56 \times 10^{5}, C_{w}=-2749$; (b) $R e=4.65 \times 10^{5}, C_{w}=-2749$; (c) $R e=9.2 \times$ $10^{5}, C_{w}=-2749$; (d) $R e=1.56 \times 10^{5}, C_{w}=-4392$; (e) $R e=1.56 \times 10^{5}$, $C_{w}=-8672$.

- Fig.8: Effect of the Prandtl number on the local Nusselt number distribution along the shroud for $R e=9.2 \times 10^{5}$ and $C_{w}=-2749$ (RSM).

- Fig.9: Effect of the Prandtl number on the averaged Nusselt number for different $\left(R e, C_{w}\right)$.

- Fig.10: Radial evolution of the local Nusselt number along the stator $\left(z^{*}=\right.$ 1): (a) influence of the coolant flowrate coefficient $C_{w}$ for $R e=1.44 \times 10^{6}$ and $\operatorname{Pr}=0.7$; (b) influence of the Reynolds number $R e$ for $C_{w}=12082$ and $\operatorname{Pr}=0.7$. Comparison between the experimental values of Djaoui et al. [2] (symbols) and the predictions of the RSM model (lines).

- Fig.11: Streamlines $\Psi^{*}=\Psi /\left(\Omega R_{2}^{2}\right)$ patterns (RSM), 30 regularly spaced intervals for $\operatorname{Pr}=0.7$ and : (a) $\operatorname{Re}=5 \times 10^{5}, C_{w}=12082,-0.128 \leq \Psi^{*} \leq$ 0.006 ; (b) $R e=10^{6}, C_{w}=12082,-0.064 \leq \Psi^{*} \leq 0.022$; (c) $R e=1.44 \times 10^{6}$, $C_{w}=12082,-0.045 \leq \Psi^{*} \leq 0.027$; (d) $R e=1.44 \times 10^{6}, C_{w}=5209$, $-0.019 \leq \Psi^{*} \leq 0.035$; (e) $R e=1.44 \times 10^{6}, C_{w}=0,0 \leq \Psi^{*} \leq 0.019$.

- Fig.12: Isotherm maps $T^{*}$ at a given Prandtl $P r=0.7$ number for various flow conditions (RSM), 30 regularly spaced intervals: (a) $R e=5 \times 10^{5}$, $C_{w}=12082$, (b) $R e=10^{6}, C_{w}=12082$, (c) $R e=1.44 \times 10^{6}, C_{w}=12082$, (d) $R e=1.44 \times 10^{6}, C_{w}=5209$, (e) $R e=1.44 \times 10^{6}, C_{w}=0$ (RSM).

- Fig.13: Temperature profiles for $C_{w}=12082, R e=1.44 \times 10^{6}$ and $\operatorname{Pr}=$ 0.7 at three radial locations: (a) $r^{*}=0.53$, (b) $r^{*}=0.69$, (c) $r^{*}=0.83$. 
Comparison between the $(\circ)$ theorical and $(\times)$ experimental values of Djaoui et $a l .[2]$ and $(-)$ the predictions of the RSM model.

- Fig.14: Effect of the Prandtl number on the local Nusselt number distribution along the stator for $R e=1.44 \times 10^{6}$ and $C_{w}=12082(\mathrm{RSM})$.

- Fig.15: (a) Effect of the Prandtl number on the averaged Nusselt number for different $\left(R e, C_{w}\right)$; (b) Validation of the correlation law (23).

- Fig.16: Velocity-temperature correlations for $R e=1.44 \times 10^{6}, \operatorname{Pr}=0.7$ and $C_{w}=12082$ at three radial locations: (a) $r^{*}=0.53$, (b) $r^{*}=0.69$, (c) $r^{*}=$ 0.83; Comparisons between (-) the RSM model and (o) the measurements of Djaoui et al. [2]. 
(a)

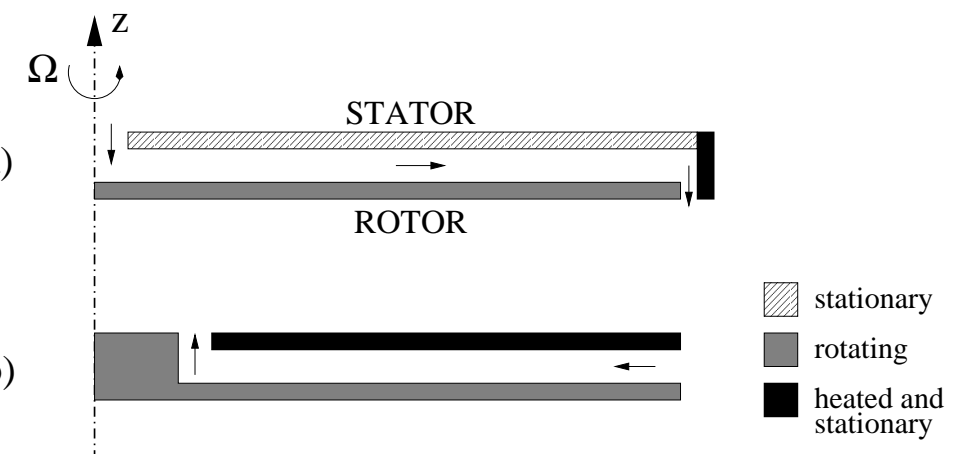

(c)

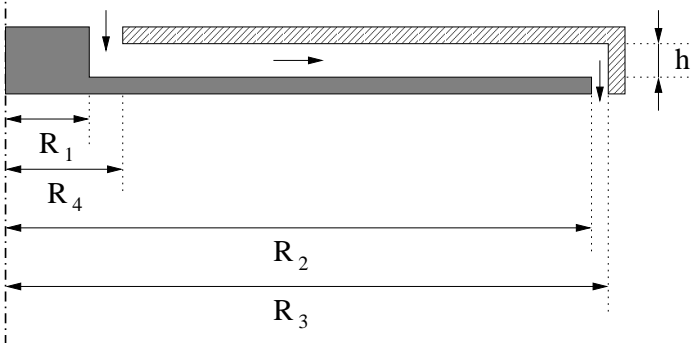

Fig. 1. Poncet S. and Schiestel R., Int. J. Heat Mass Transfer. 
(a)
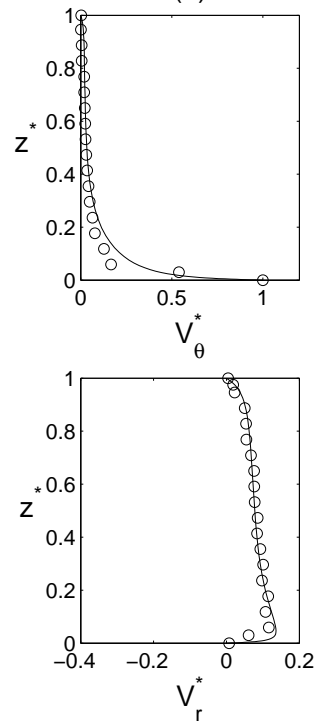

(b)
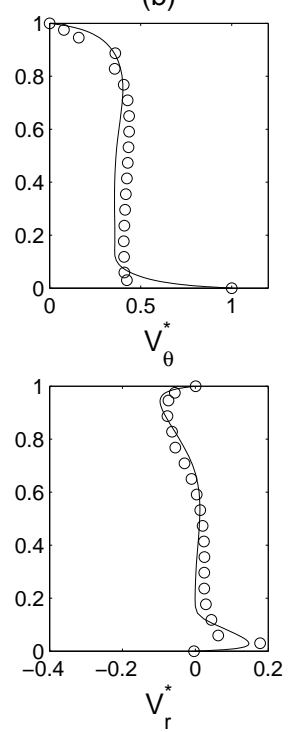

(c)
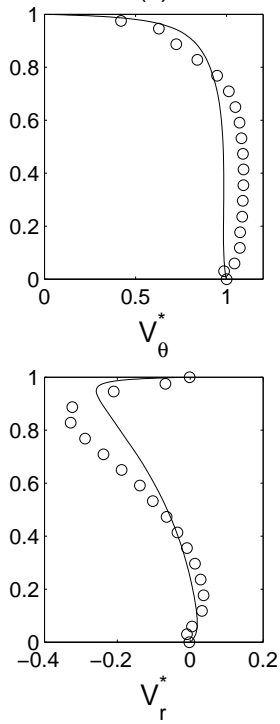

Fig. 2. Poncet S. and Schiestel R., Int. J. Heat Mass Transfer. 
(a)
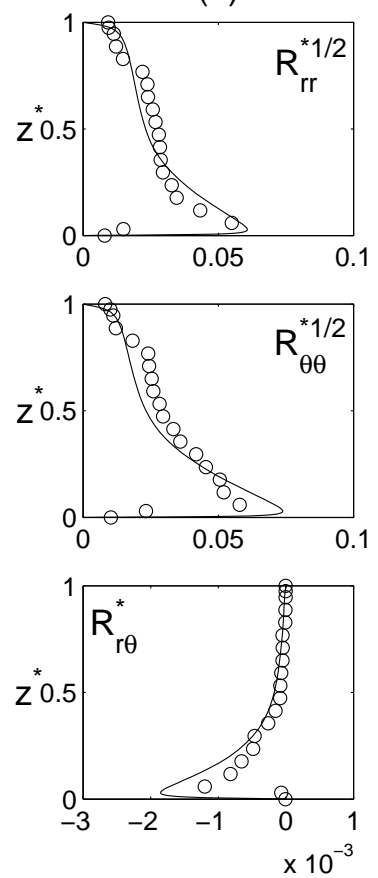

(b)
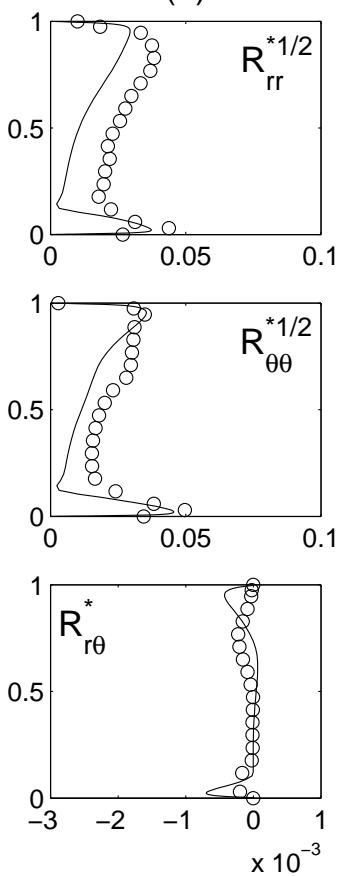

(c)
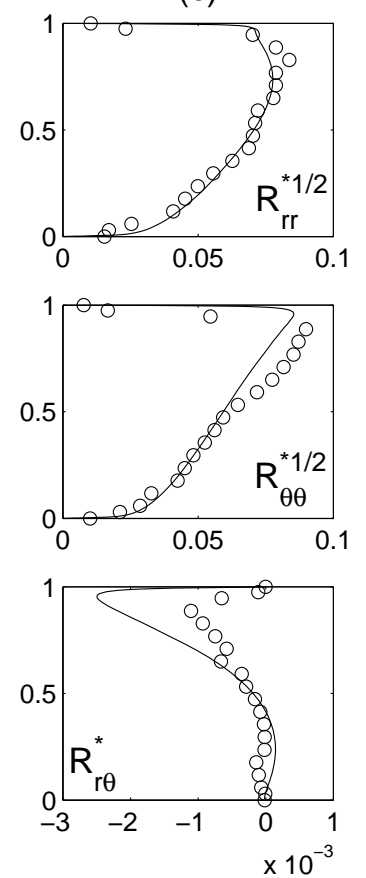

Fig. 3. Poncet S. and Schiestel R., Int. J. Heat Mass Transfer. 
(a)

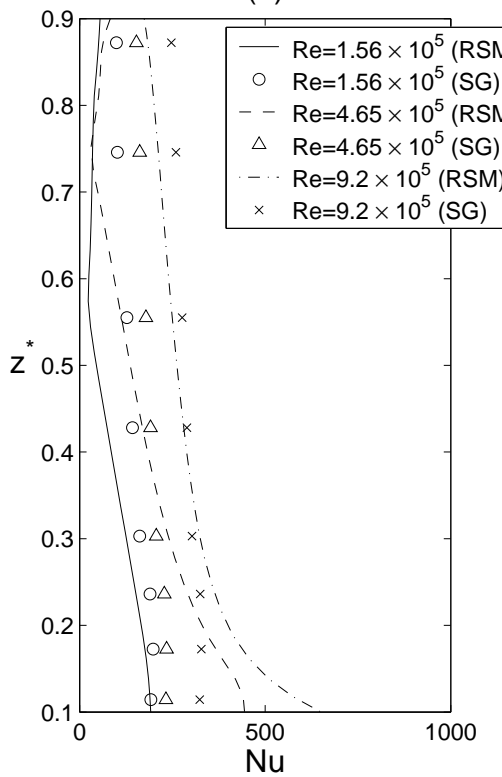

(b)

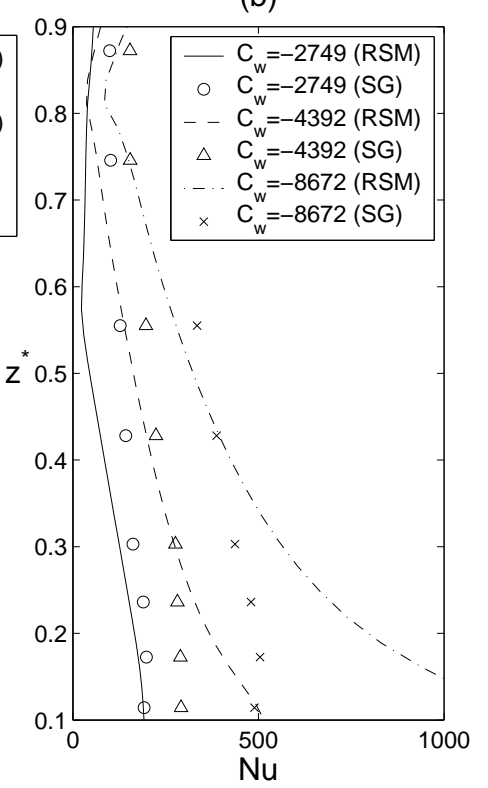

Fig. 4. Poncet S. and Schiestel R., Int. J. Heat Mass Transfer. 
(a)

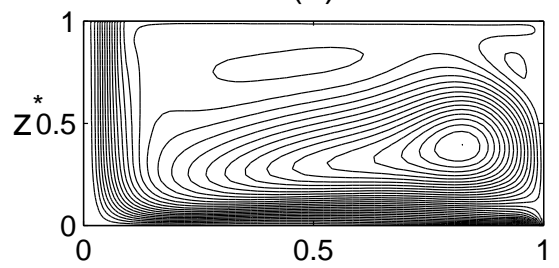

(c)

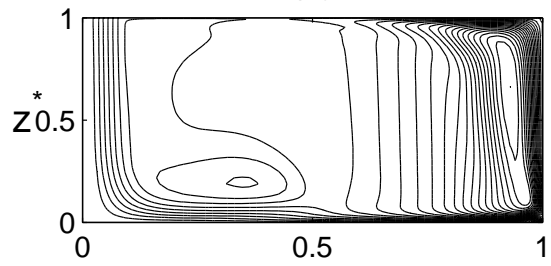

(e)

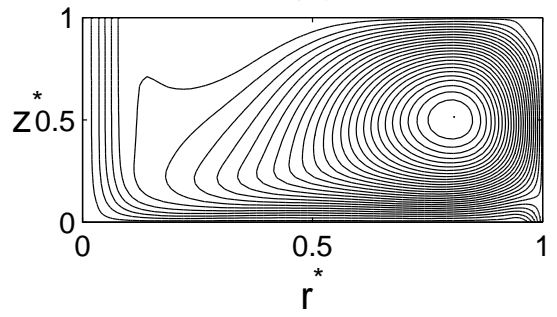

(b)

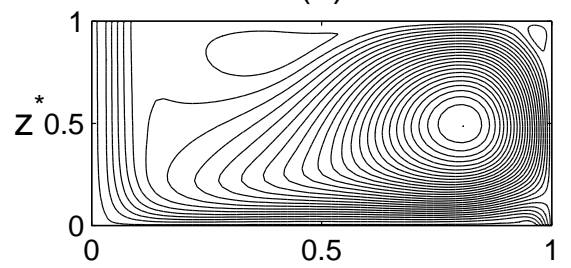

(d)

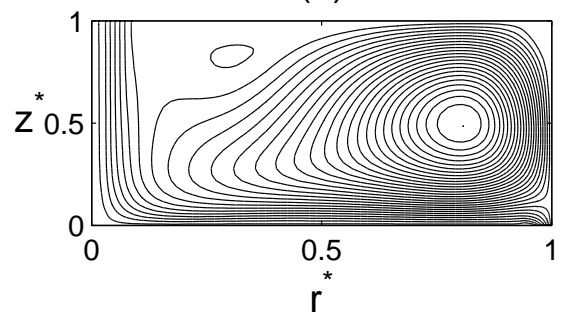

Fig. 5. Poncet S. and Schiestel R., Int. J. Heat Mass Transfer. 
(a)

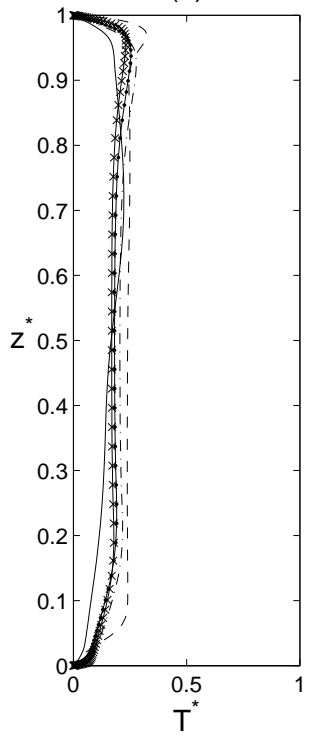

(b)

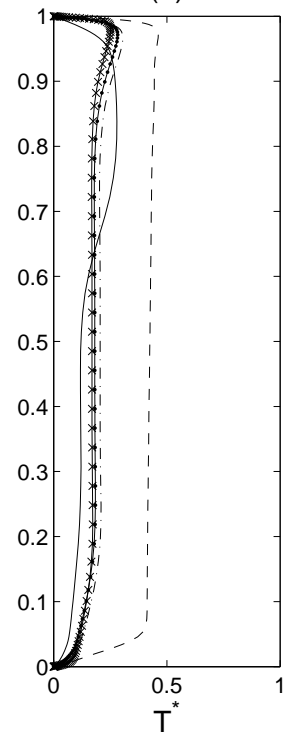

(c)

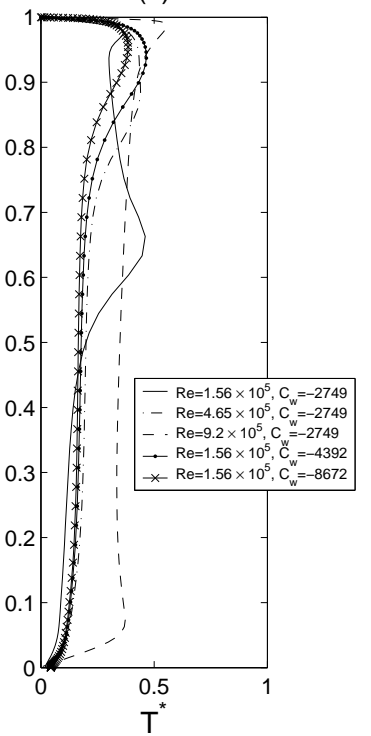

Fig. 6. Poncet S. and Schiestel R., Int. J. Heat Mass Transfer. 
(a)

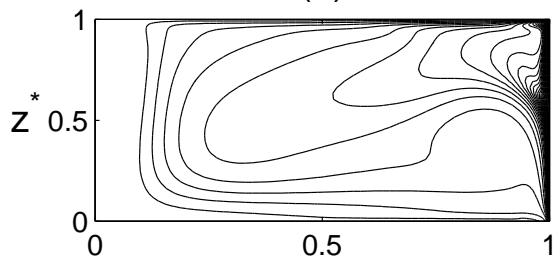

(c)

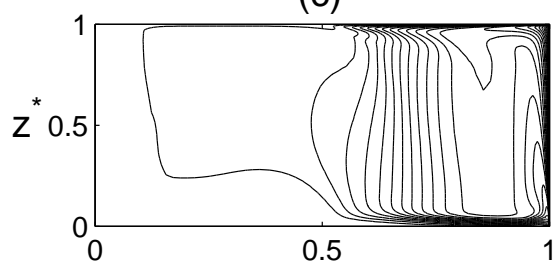

(e)

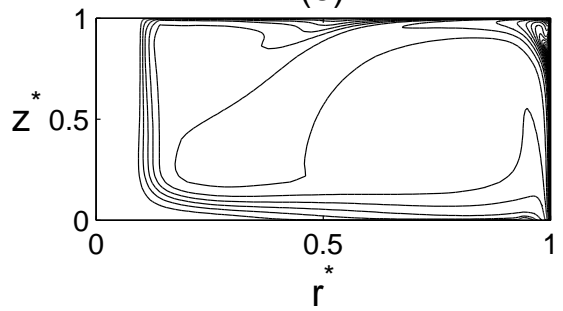

(b)

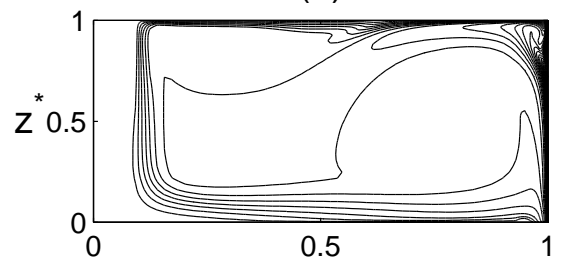

(d)

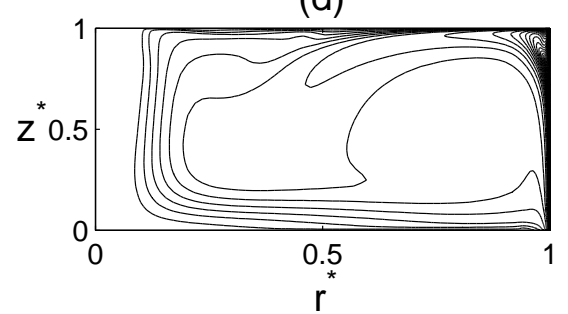

Fig. 7. Poncet S. and Schiestel R., Int. J. Heat Mass Transfer. 


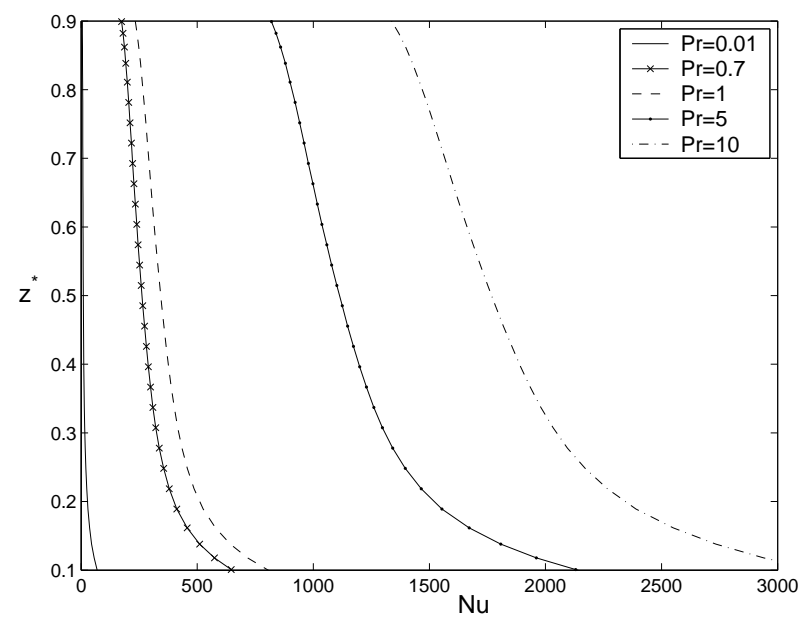

Fig. 8. Poncet S. and Schiestel R., Int. J. Heat Mass Transfer. 


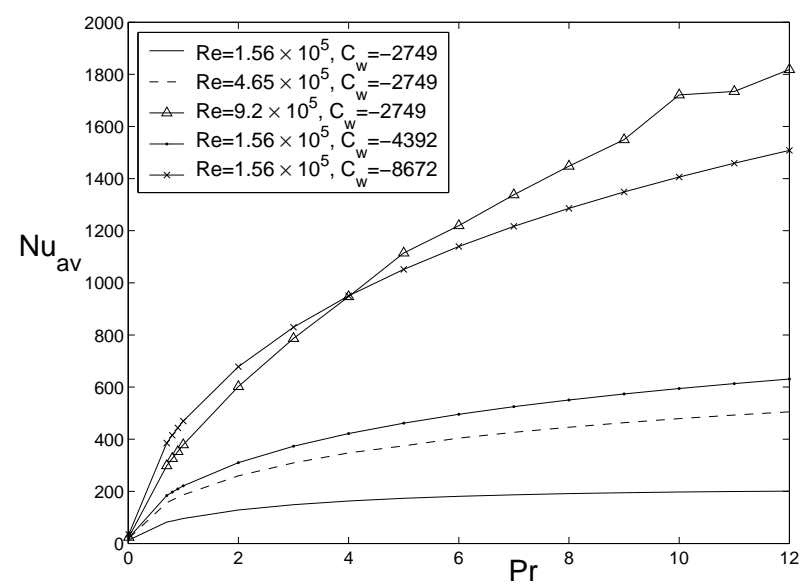

Fig. 9. Poncet S. and Schiestel R., Int. J. Heat Mass Transfer. 
(a)

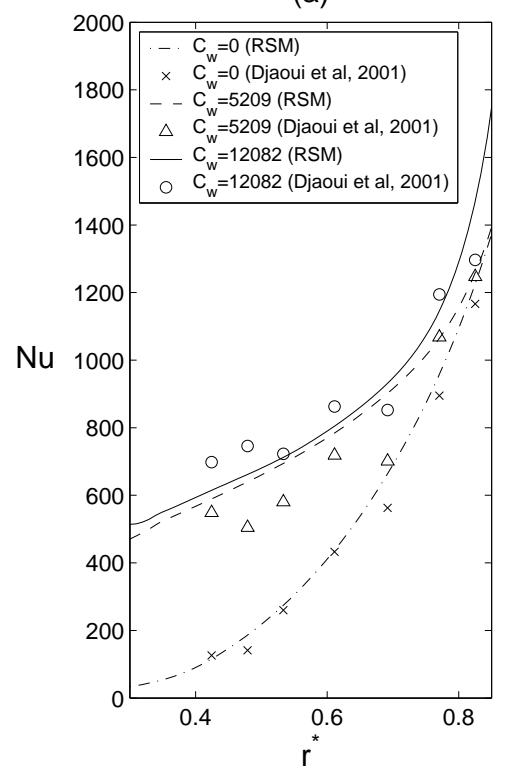

(b)

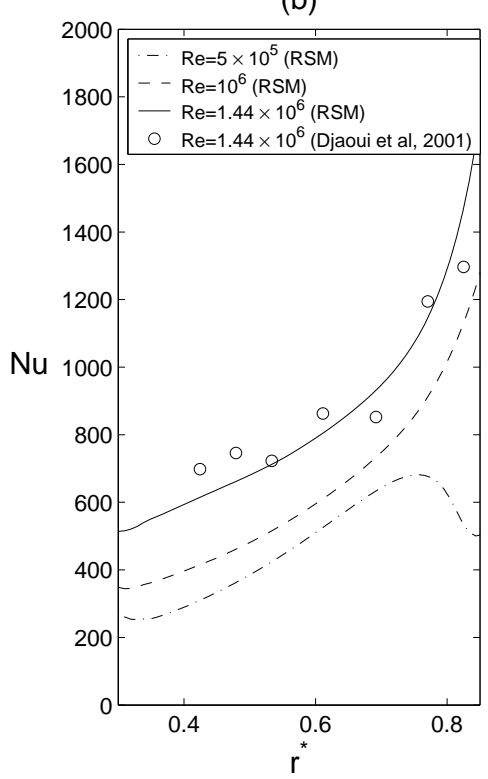

Fig. 10. Poncet S. and Schiestel R., Int. J. Heat Mass Transfer. 
(a)

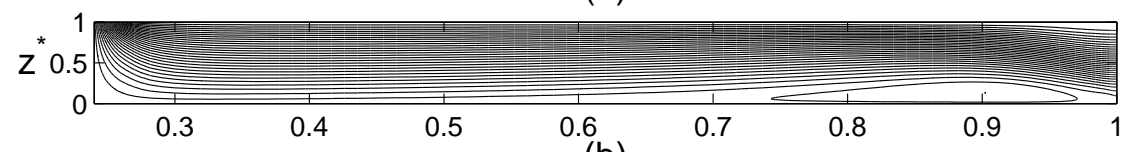

(b)

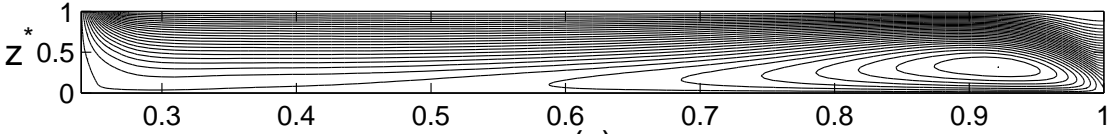

(c)

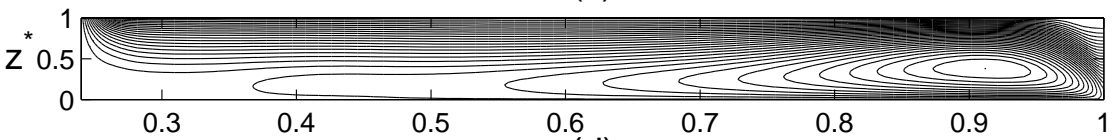

(d)

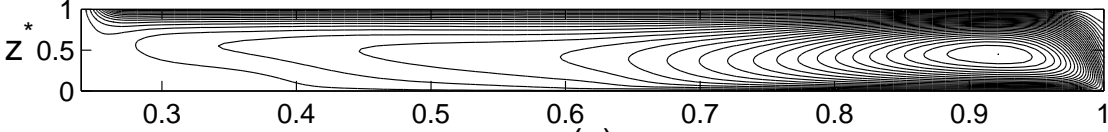

(e)

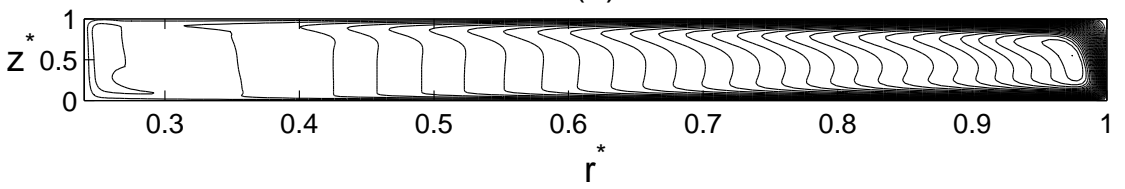

Fig. 11. Poncet S. and Schiestel R., Int. J. Heat Mass Transfer. 


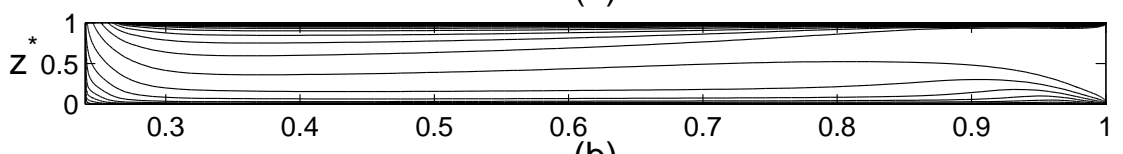

(b)

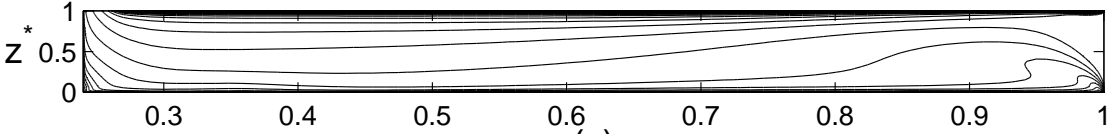

(c)

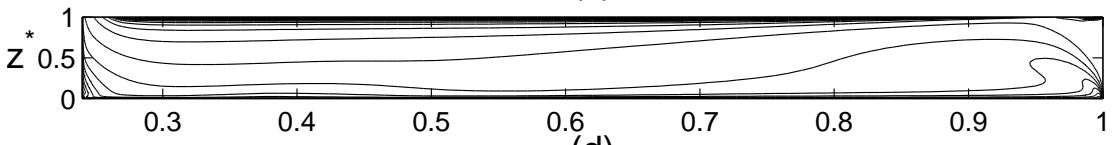

(d)

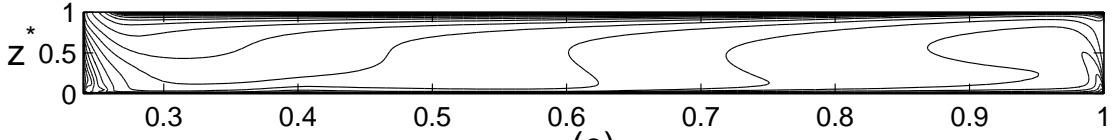

(e)

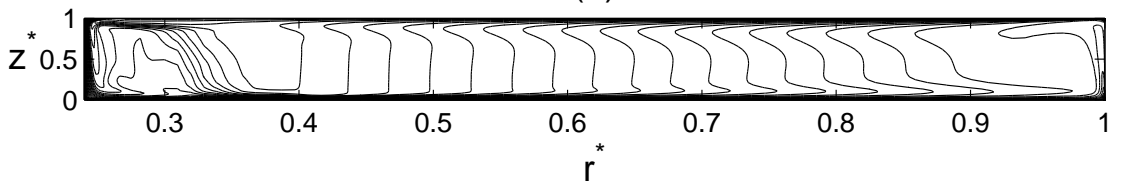

Fig. 12. Poncet S. and Schiestel R., Int. J. Heat Mass Transfer. 

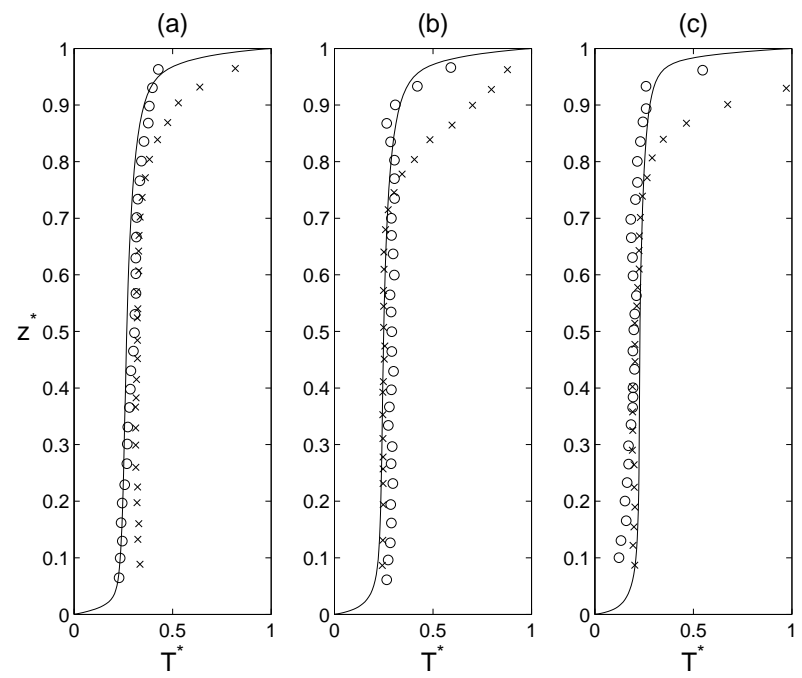

Fig. 13. Poncet S. and Schiestel R., Int. J. Heat Mass Transfer. 


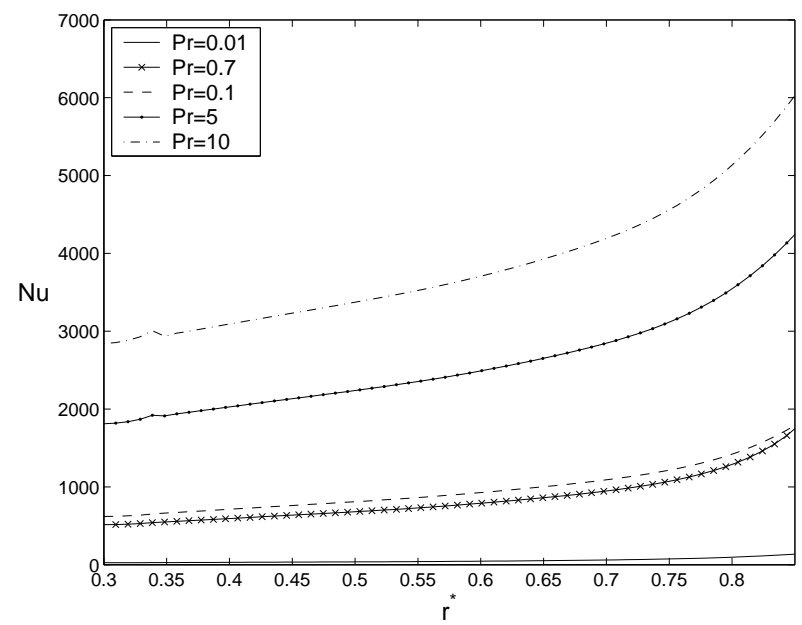

Fig. 14. Poncet S. and Schiestel R., Int. J. Heat Mass Transfer. 


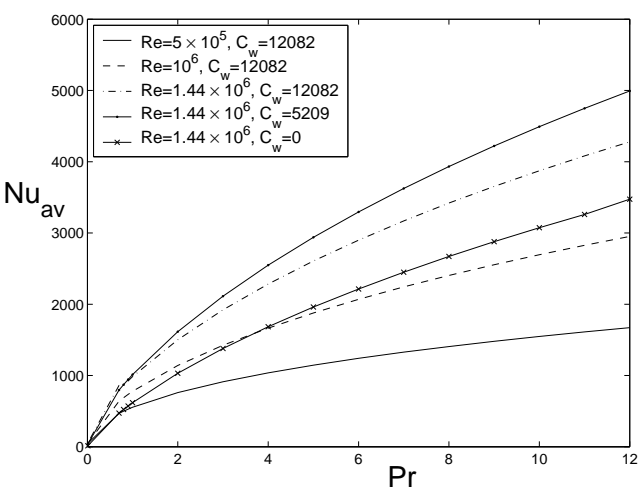

(a)

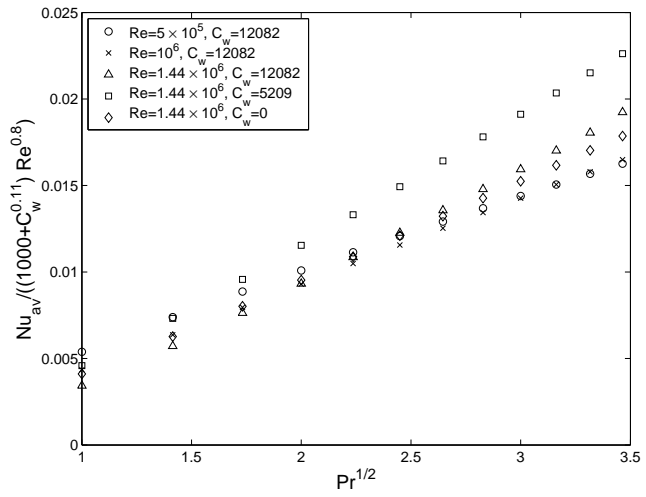

(b)

Fig. 15. Poncet S. and Schiestel R., Int. J. Heat Mass Transfer. 
(a)
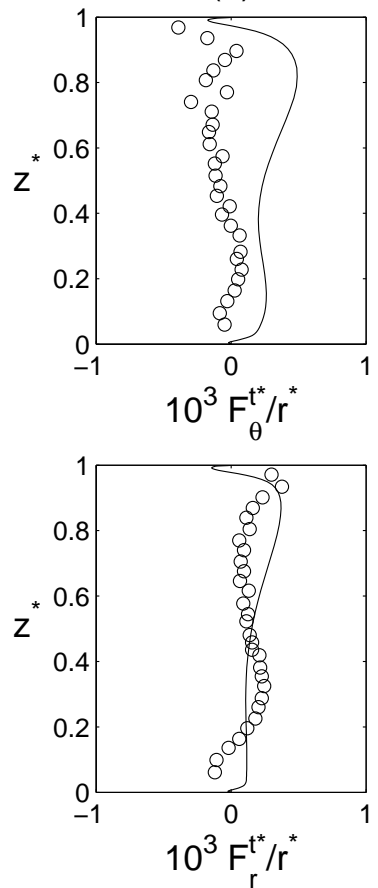

(b)
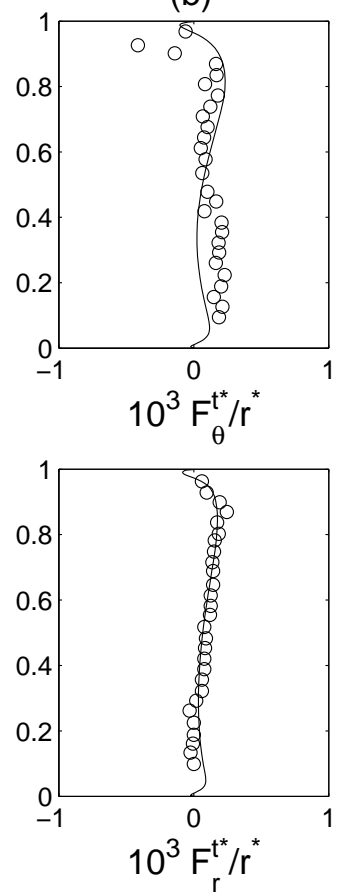

(c)
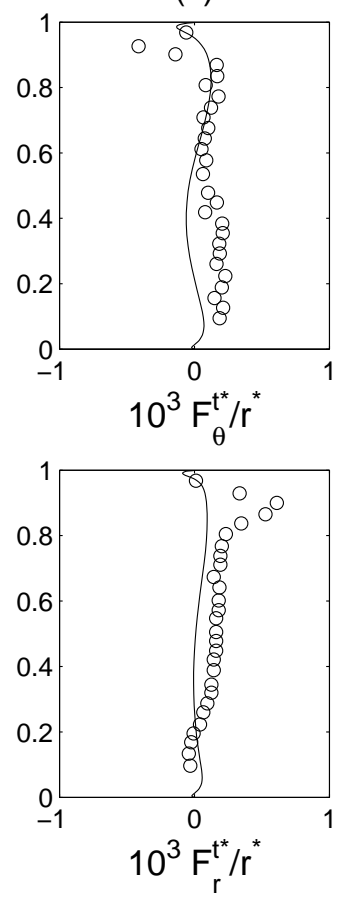

Fig. 16. Poncet S. and Schiestel R., Int. J. Heat Mass Transfer. 
- Tab.1: Characteristics of the three configurations studied.

\begin{tabular}{ccccccccccc} 
case & $R_{1}$ & $R_{2}$ & $R_{3}$ & $R_{4}$ & $h(\mathrm{~m})$ & $G$ & $R_{c}$ & $T_{0}$ & $T_{w}\left({ }^{\circ} C\right)$ & $\kappa$ \\
\hline A & 0 & 0.216 & 0.2287 & 0.0254 & 0.096 & $4 / 9$ & 1 & 22 & 97 & 0.254 \\
B & 0.09 & 0.375 & 0.375 & 0.1 & 0.03 & 0.08 & 1.632 & 24 & 59 & 0.118 \\
C & 0.038 & 0.25 & 0.253 & 0.0545 & 0.009 & 0.036 & 1.359 & 23 & 23 & 0
\end{tabular}

Table 1

Poncet S. and Schiestel R., Int. J. Heat Mass Transfer. 